Commun. Fac. Sci. Univ. Ank. Ser. A1 Math. Stat.

Volume 68, Number 1, Pages 299-325 (2019)

DOI: $10.31801 /$ cfsuasmas.415924

ISSN 1303-5991 E-ISSN 2618-6470

http://communications.science.ankara.edu.tr/index.php?series=A1

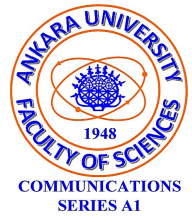

\title{
VERTICAL AND COMPLETE LIFTS OF SECTIONS OF A (DUAL) VECTOR BUNDLE AND LEGENDRE DUALITY
}

\author{
E. PEYGHAN, L. NOURMOHAMMADIFAR, AND C. M. ARCUS
}

\begin{abstract}
Using the covariant derivative for exterior forms of a (dual) vector bundle, the complete lift of an arbitrary section of a (dual) vector bundle is discovered. A theory of Legendre type and Legendre duality between vertical lifts and between complete lifts are presented. Finally, a duality between Lie algebroids structures is developed.
\end{abstract}

\section{INTRODUCTION}

The Sasaki lift of a Riemannian metric structure on $M$ is an important example of metric structures on $T M$ which is used in differential geometry with many applications in physics 19. Lifts of geometrical structures of TM to TTM were introduced and studied by several authors [8, 9, 10, 11, 20, In many papers such as [5, 12, 14, 17, 18, the authors studied the lifts of geometric objects to the second order tangent bundle, tensor bundle and jet bundle.

The Lie algebroids are important issues in physics and mechanics since the extension of Lagrangian and Hamiltonian systems to their entity [6, 7, 15] and catching the Poisson structure [16]. Several authors presented and studied the lift of geometrical objects of a Lie algebroid to the prolongation Lie algebroid. Using the vertical and complete lifts of sections of a Lie algebroid, the first author presented important results about Lie symmetry and horizontal lifts in the general framework of prolongation Lie algebroid [13].

Extending the notion of Lie algebroid from one base manifold to a pair of diffeomorphic base manifolds, the third author introduced the generalized Lie algebroid 1. Using the lift of a differentiable curve defined on the base of a generalized Lie algebroid, he developed a new theory of mechanical systems with many applications in physics [2]. The space used for developing this theory of mechanical systems is

Received by the editors: August 30, 2017, Accepted: January 01, 2018.

2010 Mathematics Subject Classification. 17A30, 17D25, 53C15, 53D05.

Key words and phrases. Vertical and complete lifts of sections of a (dual) vector bundle and Legendre duality .

(C)2018 Ankara University Communications Faculty of Sciences University of Ankara-Series A1 Mathematics and Statistics 
the Lie algebroid generalized tangent bundle

$$
\left(\left((\rho, \eta) T F,(\rho, \eta) \tau_{F}, F\right),[,]_{(\rho, \eta) T F},\left(\tilde{\rho}, I d_{F}\right)\right),
$$

of a generalized Lie algebroid $\left((F, \nu, N),[,]_{F, h},(\rho, \eta)\right)$.

The organization of the paper is as follows. Some notions and results about exterior differential algebra of a vector bundle and information about generalized Lie algebroids and in particular the Lie algebroid generalized tangent bundles

$$
\left(\left((\rho, \eta) T E,(\rho, \eta) \tau_{E}, E\right),[,]_{(\rho, \eta) T E},\left(\tilde{\rho}, I d_{E}\right)\right),
$$

and

$$
\left.\left(\left((\rho, \eta) T \stackrel{*}{E},(\rho, \eta) \tau_{E}^{*}, \stackrel{*}{E}\right),[,]_{(\rho, \eta) T E}, \stackrel{*}{\tilde{\rho}}, I d_{E}^{*}\right)\right),
$$

are recalled in Section 2. In Section 3, we introduce a Lie covariant derivative for the exterior algebra of the vector bundle $(E, \pi, M)$ and using it, we introduce in Theorem 11 the complete $(g, h)$-lift $u^{c} \in \Gamma\left(T E, \tau_{E}, E\right)$ of an arbitrary section $u \in \Gamma(E, \pi, M)$. Using the complete $(g, h)$-lift of a function $f \in \mathcal{F}(N)$ we obtain new results for vertical and complete $(g, h)$-lifts. In the final of Section 3, we introduced the complete and vertical $(g, h)$-lifts

$$
u^{C}, u^{V} \in \Gamma\left((\rho, \eta) T E,(\rho, \eta) \tau_{E}, E\right),
$$

of a section $u \in \Gamma(E, \pi, M)$ and important results are presented. A dual theory for the vertical and complete lifts is presented in Section 4 and similar results are obtained. A general presentation of Lagrange (Finsler) and Hamilton (Cartan) fundamental functions and a detailed theory of Legendre type are presented in Section 5. Using the tangent $(\rho, \eta)$-application of the Legendre bundle morphism associated to a Lagrange respectively Hamilton fundamental function, we obtain new results about duality between vertical lifts and between complete lifts in Section 5. New results about duality between Lie algebroids structures and the Legendre $(\rho, \eta)$-equivalence between the vector bundle $(E, \pi, M)$ and its dual $(\stackrel{*}{E}, \stackrel{*}{\pi}, M)$ are presented in Section 6 .

\section{Preliminaries}

Let $(E, \pi, M)$ be an arbitrary vector bundle, $\Gamma(E, \pi, M)$ be the set of the sections of the vector bundle $(E, \pi, M)$ and $\mathcal{F}(M)$ be the set of differentiable real-valued functions on $M$. For any $q \in \mathbb{N}$, we denote by $\left(\Sigma_{q}, \circ\right)$ the permutations group of $\{1,2, \ldots, q\}$. We denoted by $\Lambda^{q}(E, \pi, M)$ the set of $q$-linear applications

$$
\begin{array}{ccc}
\Gamma(E, \pi, M)^{q} & \stackrel{\omega}{\mathcal{F}}(M) \\
\left(z_{1}, \cdot, z_{q}\right) & \longmapsto & \omega\left(z_{1}, \cdot, z_{q}\right)
\end{array},
$$

such that

$$
\omega\left(z_{\sigma(1)}, \cdot, z_{\sigma(q)}\right)=\operatorname{sgn}(\sigma) \cdot \omega\left(z_{1}, \cdot, z_{q}\right),
$$

for any $z_{1}, \ldots, z_{q} \in \Gamma(E, \pi, M)$ and for any $\sigma \in \Sigma_{q}$. The elements of $\Lambda^{q}(E, \pi, M)$ will be called differential forms of degree $q$ or differential $q$-forms. 
A generalized Lie algebroid is a vector bundle $(F, \nu, N)$ given by the diagrams:

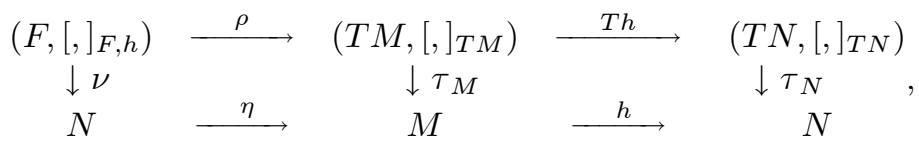

where $h$ and $\eta$ are arbitrary isomorphisms, $(\rho, \eta)$ is a vector bundles morphism from $(F, \nu, N)$ to $\left(T M, \tau_{M}, M\right)$ and the operation

$$
\begin{array}{ccc}
\Gamma(F, \nu, N) \times \Gamma(F, \nu, N) & \stackrel{[]_{F, h}}{\longrightarrow} & \Gamma(F, \nu, N) \\
(u, v) & \longmapsto & {[u, v]_{F, h}}
\end{array}
$$

satisfies in

$$
[u, f \cdot v]_{F, h}=f[u, v]_{F, h}+\Gamma(T h \circ \rho, h \circ \eta)(u) f \cdot v, \quad \forall f \in \mathcal{F}(N),
$$

such that the 4 -tuple $\left(\Gamma(F, \nu, N),+, \cdot,[,]_{F, h}\right)$ is a Lie $\mathcal{F}(N)$-algebra, where

$$
\Gamma(T h \circ \rho, h \circ \eta)\left(z^{\alpha} t_{\alpha}\right)(f)=z^{\alpha} \rho_{\alpha}^{i}\left(\frac{\partial(f \circ h)}{\partial x^{i}}\right) \circ h^{-1},
$$

for any $z^{\alpha} t_{\alpha} \in \Gamma(F, \nu, N)$ and $f \in \mathcal{F}(N)$ (see [1, 2, 3, 4, for more details). We denote by $\left((F, \nu, N),[,]_{F, h},(\rho, \eta)\right)$ the generalized Lie algebroid defined in the above. Moreover, the couple $\left([,]_{F, h},(\rho, \eta)\right)$ is called the generalized Lie algebroid structure. In particular, if $\eta=I d_{M}=h$, then we obtain the definition of Lie algebroid. So, any Lie algebroid can be regarded as a generalized Lie algebroid.

If we take local coordinates $\left(x^{i}\right)$ and $\left(\chi^{\tilde{\imath}}\right)$ on open sets $V \subset M$ and $W \subset N$, respectively, then we have the corresponding local coordinates $\left(x^{i}, y^{i}\right)$ and $\left(\chi^{\tilde{\imath}}, z^{\tilde{\imath}}\right)$ on $T M$ and $T N$, respectively, where $i, \tilde{\imath} \in\{1, \ldots, m\}$. Moreover, we consider $\left(\chi^{\tilde{\imath}}, z^{\alpha}\right)$ as the local coordinates on $F$, where $\alpha \in\{1, \ldots, p\}$. If $\left\{t_{\alpha}\right\}$ is a local basis for module of sections of $(F, \nu, N)$, then we put $\left[t_{\alpha}, t_{\beta}\right]_{F, h}=L_{\alpha \beta}^{\gamma} t_{\gamma}$, where $L_{\alpha \beta}^{\gamma}$ are local functions on $N$ and $\alpha, \beta, \gamma \in\{1, \ldots, p\}$. It is easy to see that $L_{\alpha \beta}^{\gamma}=-L_{\beta \alpha}^{\gamma}$ and

$$
\left(L_{\alpha \beta}^{\gamma} \circ h\right)\left(\rho_{\gamma}^{k} \circ h\right)=\left(\rho_{\alpha}^{i} \circ h\right) \frac{\partial\left(\rho_{\beta}^{k} \circ h\right)}{\partial x^{i}}-\left(\rho_{\beta}^{j} \circ h\right) \frac{\partial\left(\rho_{\alpha}^{k} \circ h\right)}{\partial x^{j}} .
$$

The local functions $L_{\alpha \beta}^{\gamma}$ introduced in the above are called the structure functions of the generalized Lie algebroid $\left((F, \nu, N),[,]_{F, h},(\rho, \eta)\right)$.

A morphism from $\left((F, \nu, N),[,]_{F, h},(\rho, \eta)\right)$ to $\left(\left(F^{\prime}, \nu^{\prime}, N^{\prime}\right),[,]_{F^{\prime}, h^{\prime}},\left(\rho^{\prime}, \eta^{\prime}\right)\right)$ is a morphism $\left(\varphi, \varphi_{0}\right)$ from $(F, \nu, N)$ to $\left(F^{\prime}, \nu^{\prime}, N^{\prime}\right)$ such that $\varphi_{0}$ is an isomorphism from $N$ to $N^{\prime}$, and the modules morphism $\Gamma\left(\varphi, \varphi_{0}\right)$ is a Lie algebras morphism from $\left(\Gamma(F, \nu, N),[,]_{F, h}\right)$ to $\left(\Gamma\left(F^{\prime}, \nu^{\prime}, N^{\prime}\right),[,]_{F^{\prime}, h^{\prime}}\right)$. Thus, we can discuss about the category of generalized Lie algebroids [1, 2].

2.1. The generalized tangent bundle of a vector bundle and its dual. Let $\left((F, \nu, N),[,]_{F, h},(\rho, \eta)\right)$ be a generalized Lie algebroid, $(E, \pi, M)$ be a vector 
bundle and $(g, h)$ be a vector bundle morphism from $(E, \pi, M)$ to $(F, \nu, N)$ with components $g_{b}^{\alpha}, \alpha \in\{1,2, \cdots, n\}$ and $b \in\{1,2, \cdots, r\}$. We consider the diagrams:

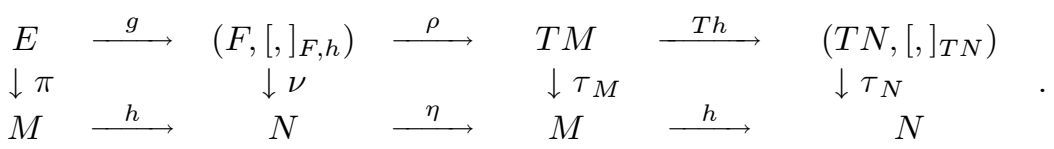

It is known that

$$
\left(\left((h \circ \pi)^{*} F,(h \circ \pi)^{*} \nu, E\right),[,]_{(h \circ \pi)^{*} F},\left(\stackrel{(h \circ \pi)^{*} F}{\rho}, I d_{E}\right)\right),
$$

is a Lie algebroid which is called the pull-back Lie algebroid of the generalized Lie algebroid $\left((F, \nu, N),[,]_{F, h},(\rho, \eta)\right)$, where $\stackrel{(h \circ \pi)^{*} F}{\rho}:(h \circ \pi)^{*} F \rightarrow T E$ is defined by

$$
\stackrel{(h \circ \pi)^{*} F}{\rho}\left(X^{\alpha} T_{\alpha}\right)=\left(g_{b}^{\alpha} \circ \pi\right)\left(u^{b} \circ \pi\right)\left(\rho_{\alpha}^{i} \circ h \circ \pi\right) \partial_{i},
$$

and $[,]_{(h \circ \pi)^{*} F}$ is given by

$$
\begin{aligned}
& {\left[T_{\alpha}, f T_{\beta}\right]_{(h \circ \pi)^{*} F}=f\left(L_{\alpha \beta}^{\gamma} \circ h \circ \pi\right) T_{\gamma}+\left(\rho_{\alpha}^{i} \circ h \circ \pi\right) \partial_{i}(f) T_{\beta},} \\
& {\left[f T_{\alpha}, T_{\beta}\right]_{(h \circ \pi)^{*} F}=-\left[T_{\beta}, f T_{\alpha}\right]_{(h \circ \pi)^{*} F},}
\end{aligned}
$$

for any $f \in \mathcal{F}(E)$. Let $\left(\partial_{i}, \dot{\partial}_{a}\right)$ be the base sections for the Lie $\mathcal{F}(E)$-algebra $\Gamma\left(T E, \tau_{E}, E\right)$ and we consider

$$
\tilde{\partial}_{\alpha}=T_{\alpha} \oplus\left(\rho_{a}^{i} \circ h \circ \pi\right) \partial_{i}, \quad \dot{\tilde{\partial}}_{a}=0_{(h \circ \pi)^{*} F} \oplus \dot{\partial}_{a} .
$$

Now, we consider the vector subbundle $\left((\rho, \eta) T E,(\rho, \eta) \tau_{E}, E\right)$ of the vector bundle $\left((h \circ \pi)^{*} F \oplus T E, \stackrel{\oplus}{\pi}, E\right)$, for which the $\mathcal{F}(E)$-module of its sections is the $\mathcal{F}(E)$ submodule of $\Gamma\left((h \circ \pi)^{*} F \oplus T E, \stackrel{\oplus}{\pi}, E\right)$ generated by the set of sections $\left(\tilde{\partial}_{\alpha}, \tilde{\tilde{\partial}}_{a}\right)$. The base sections $\left(\tilde{\partial}_{\alpha}, \dot{\tilde{\partial}}_{a}\right)$ are called the natural $(\rho, \eta)$-base. Defining

$$
\tilde{\rho}\left(X^{\alpha} \tilde{\partial}_{\alpha}+\tilde{X}^{a} \dot{\tilde{\partial}}_{a}\right)=X^{\alpha}\left(\rho_{\alpha}^{i} \circ h \circ \pi\right) \partial_{i}+\tilde{X}^{a} \dot{\partial}_{a},
$$

and

$$
\begin{array}{r}
{\left[X_{1}^{\alpha} \tilde{\partial}_{\alpha}+\tilde{X}_{1}^{a} \dot{\tilde{\partial}}_{a}, X_{2}^{\beta} \tilde{\partial}_{\beta}+\tilde{X}_{2}^{b} \dot{\tilde{\partial}}_{b}\right]_{(\rho, \eta) T E}=\left[X_{1}^{\alpha} T_{\alpha}, X_{2}^{\beta} T_{\beta}\right]_{(h \circ \pi)^{*} F}} \\
\oplus\left[\left(X_{1}^{\alpha}\left(\rho_{\alpha}^{i} \circ h \circ \pi\right) \partial_{i}+\tilde{X}_{1}^{a} \dot{\partial}_{a}, X_{2}^{\beta}\left(\rho_{\beta}^{j} \circ h \circ \pi\right) \partial_{j}+\tilde{X}_{2}^{b} \dot{\partial}_{b}\right)\right]_{T E},
\end{array}
$$

easily we obtain Lie algebroid structure $\left([,]_{(\rho, \eta) T E},\left(\tilde{\rho}, I d_{E}\right)\right)$ for $\left((\rho, \eta) T E,(\rho, \eta) \tau_{E}, E\right)$ which is called the generalized tangent bundle (see 1, 2, for more details). Similarly, we consider the diagram

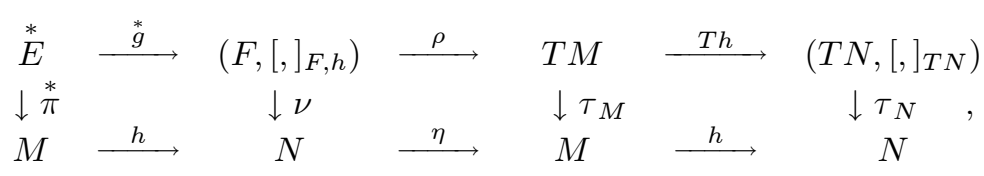


where $(\stackrel{*}{g}, h)$ is a vector bundle morphism from $(\stackrel{*}{E}, \stackrel{*}{\pi}, M)$ to $(F, \nu, N)$ with components $g^{\alpha b}, \alpha \in\{1,2, . ., n\}$ and $b \in\{1,2, . ., r\}$. Let $\left(\stackrel{*}{\partial}_{i}, \dot{\partial}^{a}\right)$ be the base sections for the Lie $\mathcal{F}(\stackrel{*}{E})$-algebra $\left(\Gamma\left(T \stackrel{*}{E}, \tau_{E}^{*}, \stackrel{*}{E}\right),[,]_{T E}\right)$. Defining

$$
(h \circ \stackrel{*}{\pi})^{*} F\left(X^{\alpha} \stackrel{*}{T}_{\alpha}\right)=\left(g^{\alpha b} \circ \stackrel{*}{\pi}\right)\left(u_{b} \circ \stackrel{*}{\pi}\right)\left(\rho_{\alpha}^{i} \circ h \circ \stackrel{*}{\pi}\right) \stackrel{*}{\partial}_{i},
$$

and

$$
\begin{aligned}
& {\left[\stackrel{*}{T}_{\alpha}, f \stackrel{*}{T}_{\beta}\right]_{(h \circ *) * F}=f\left(L_{\alpha \beta}^{\gamma} \circ h \circ \stackrel{*}{\pi}\right) \stackrel{*}{T}_{\gamma}+\left(\rho_{\alpha}^{i} \circ h \circ \stackrel{*}{\pi}\right) \stackrel{*}{\partial}_{i}(f) \stackrel{*}{T}_{\beta},} \\
& {\left[f \stackrel{*}{T}_{\alpha}, \stackrel{*}{T}_{\beta}\right]_{(h \circ *) * F}=-\left[\stackrel{*}{T}_{\beta}, f \stackrel{*}{T}_{\alpha}\right]_{(h \circ *)^{*} F},}
\end{aligned}
$$

it is easy to check that

$$
\left(\left((h \circ \stackrel{*}{\pi})^{*} F,(h \circ \stackrel{*}{\pi})^{*} \nu, \stackrel{*}{E}\right),[,]_{(h \circ \pi)^{*} F},\left(\stackrel{\left(h \circ \frac{*}{\pi}\right)^{*} F}{\rho}, I d_{E}^{*}\right)\right),
$$

is a Lie algebroid which is called the pull-back Lie algebroid of $\left((F, \nu, N),[,]_{F, h},(\rho, \eta)\right)$ [3]. Now, we consider the vector subbundle $\left((\rho, \eta) T \stackrel{*}{E},(\rho, \eta) \tau_{E}^{*}, \stackrel{*}{E}\right)$ of the vector bundle $\left((h \circ \stackrel{*}{\pi})^{*} F \oplus T \stackrel{*}{E}, \stackrel{\oplus}{\pi}, \stackrel{*}{E}\right)$, such that the $\mathcal{F}(\stackrel{*}{E})$-module of its sections is generated by the set of sections $\left(\tilde{\partial}_{\alpha}^{*}, \dot{\tilde{\partial}}^{a}\right)$, where

$$
\stackrel{*}{\partial}_{\alpha}=\stackrel{*}{T}_{\alpha} \oplus\left(\rho_{\alpha}^{i} \circ h \circ \stackrel{*}{\pi}\right) \stackrel{*}{\partial}_{i}, \quad \dot{\tilde{\partial}}^{a}=0_{(h \circ *)^{*} F} \oplus \dot{\partial}^{a}
$$

Considering

$$
\stackrel{*}{\rho}^{(}\left(X^{\alpha} \stackrel{*}{\partial}_{\alpha}+\tilde{X}_{a} \dot{\tilde{\partial}}^{a}\right)=X^{\alpha}\left(\rho_{\alpha}^{i} \circ h \circ *{ }^{*}\right) \stackrel{*}{\partial}_{i}+\tilde{X}_{a} \dot{\partial}^{a}
$$

and

$$
\begin{aligned}
& \left.\left[X_{1}^{\alpha}{\stackrel{*}{\tilde{\partial}_{\alpha}}}_{\alpha}+\tilde{X}_{a}^{1} \dot{\tilde{\partial}}^{a}, X_{2}^{\beta} \stackrel{*}{\tilde{\partial}}_{\beta}+\tilde{X}_{b}^{2} \dot{\tilde{\partial}}^{b}\right]_{(\rho, \eta) T E}{ }^{*}=\left[X_{1}^{\alpha} \stackrel{T}{\alpha}_{\alpha}+X_{2}^{\beta} T_{\beta}^{*}\right]_{(h \circ \pi}\right)^{*}{ }_{F} \\
& \oplus\left[X_{1}^{\alpha}\left(\rho_{\alpha}^{i} \circ h \circ \stackrel{*}{\pi}\right) \stackrel{*}{\partial}_{i}+\tilde{X}_{a}^{1} \dot{\partial}^{a}, X_{2}^{\beta}\left(\rho_{\beta}^{j} \circ h \circ \stackrel{*}{\pi}\right) \stackrel{*}{\partial}_{j}+\tilde{X}_{b}^{2} \dot{\partial}^{b}\right]_{T E}^{*},
\end{aligned}
$$

easily we deduce that $\left.\left([,]_{(\rho, \eta) T E}, \stackrel{*}{\stackrel{*}{\rho}}, I d_{E}^{*}\right)\right)$ is a Lie algebroid structure for $\left((\rho, \eta) T \stackrel{*}{E},(\rho, \eta) \tau_{E}^{*}, \stackrel{*}{E}\right)$ which is called the generalized tangent bundle (see [1, 2], for more details).

\section{Vertical and complete $(g, h)$-Lifts of Sections of a Vector Bundle}

In this section, we consider the diagram 2.2 for the generalized Lie algebroid $\left((F, \nu, N),[,]_{F, h},(\rho, \eta)\right)$. Also, we admit that $(g, h)$ is a vector bundles morphism locally invertible from $(E, \pi, M)$ to $(F, \nu, N)$ with components

$$
g_{b}^{\alpha}, \quad \alpha \in\{1, \cdots, n\}, \quad b \in\{1, \ldots, r\} .
$$


So, for any vector local $(m+r)$-chart $\left(V, t_{V}\right)$ of $(E, \pi, M)$, there exist the real functions

$$
V \stackrel{\tilde{g}_{\alpha}^{b}}{\longrightarrow} \mathbb{R}, \quad \alpha \in\{1, \cdots, n\}, \quad b \in\{1, \cdots, r\},
$$

such that $\tilde{g}_{\alpha}^{b}(\varkappa) \cdot g_{a}^{\alpha}(\varkappa)=\delta_{a}^{b}$ and $\tilde{g}_{\alpha}^{a}(\varkappa) \cdot g_{a}^{\beta}(\varkappa)=\delta_{\alpha}^{\beta}$, for any $\varkappa \in V$. Thus, we can discuss about vector bundles morphism $\left(g^{-1}, h^{-1}\right)$ from $(F, \nu, N)$ to $(E, \pi, M)$ with components

$$
\tilde{g}_{\alpha}^{b} \circ h^{-1}, \alpha \in\{1, \cdots, n\}, \quad b \in\{1, \cdots, r\} .
$$

Definition 3.1. If $f \in \mathcal{F}(N)$ (respectively $f \in \mathcal{F}(M)$ ), then the real function $f^{\vee}=f \circ h \circ \pi$ (respectively $f^{\vee}=f \circ \pi$ ) is called the vertical lift of the functionf.

It is remarkable that since

$$
\left(\Gamma(T h \circ \rho, h \circ \eta) \Gamma(g, h)\left(u^{a} s_{a}\right)\right)(f)=\left(g_{b}^{\alpha} u^{b}\right) \circ h^{-1} \rho_{\alpha}^{i} \frac{\partial(f \circ h)}{\partial x^{i}} \circ h^{-1},
$$

then using the above definition we get

$$
\left(\Gamma(T h \circ \rho, h \circ \eta) \Gamma(g, h)\left(u^{a} s_{a}\right)(f)\right)^{v}=\left(\left(g_{b}^{\alpha} u^{b} \rho_{\alpha}^{i} \circ h\right) \circ \pi\right) \partial_{i}(f \circ h \circ \pi) .
$$

Definition 3.2. If $u=u^{a} s_{a}$ is a section of $(E, \pi, M)$, then we introduce the vertical lift of $u$ as section of $\Gamma\left(T E, \tau_{E}, E\right)$ given by

$$
u^{\vee}=\left(u^{b} \circ \pi\right) \dot{\partial}_{a} .
$$

If $\left\{s_{a}\right\}$ be a basis of sections of $\Gamma(E, \pi, M)$, then using the above equation we have

$$
s_{a}^{\vee}=\dot{\partial}_{a} .
$$

Using the locally expression of $u^{\vee}$ we can deduce

Lemma 3.3. If $u$ and $v$ are sections of $E$ and $f \in \mathcal{F}(M)$, then

$$
(u+v)^{\vee}=u^{\vee}+v^{\vee}, \quad(f u)^{\vee}=f^{\vee} u^{\vee}, \quad u^{\vee}\left(f^{\vee}\right)=0 .
$$

For any $z \in \Gamma(F, \nu, N)$, the $\mathcal{F}(N)$-multilinear application

$$
\Lambda(F, \nu, N) \stackrel{L_{z}}{\longrightarrow} \Lambda(F, \nu, N),
$$

defined by

$$
L_{z}(f)=[\Gamma(T h \circ \rho, h \circ \eta) z](f), \forall f \in \mathcal{F}(N),
$$

and

$$
\begin{aligned}
L_{z} \theta\left(z_{1}, \ldots, z_{q}\right) & =\Gamma(T h \circ \rho, h \circ \eta) z\left(\omega\left(z_{1}, \ldots, z_{q}\right)\right) \\
& -\sum_{i=1}^{q} \theta\left(\left(z_{1}, \ldots,\left[z, z_{i}\right]_{F, h}, \ldots, z_{q}\right)\right),
\end{aligned}
$$

for any $\theta \in \Lambda^{q}(F, \nu, N)$ and $z_{1}, \ldots, z_{q} \in \Gamma(F, \nu, N)$, will be called the covariant Lie derivative with respect to the section $z$. Also for any $u \in \Gamma(E, \pi, M)$, the $\mathcal{F}(M)$ multilinear application

$$
\Lambda(E, \pi, M) \stackrel{(g, h) \mathcal{L}_{u}}{\longrightarrow} \Lambda(E, \pi, M),
$$


defined by

$$
(g, h) \mathcal{L}_{u}(f)=(g, h)^{*}\left\{\Gamma(T h \circ \rho, h \circ \eta)(\Gamma(g, h) u)\left(g^{-1}, h^{-1}\right)^{*} f\right\}, \forall f \in \mathcal{F}(M),
$$

and

$$
\begin{aligned}
& (g, h) \mathcal{L}_{u} \omega\left(u_{1}, \ldots, u_{q}\right) \\
& =(g, h)^{*}\left\{\mathcal{L}_{\Gamma(g, h) u}\left(g^{-1}, h^{-1}\right)^{*} \omega\left(\Gamma(g, h) u_{1}, \ldots, \Gamma(g, h) u_{q}\right)\right\} \\
& =(g, h)^{*}\left\{\Gamma(T h \circ \rho, h \circ \eta)[\Gamma(g, h) u]\left(g^{-1}, h^{-1}\right)^{*} \omega\left(\Gamma(g, h) u_{1}, \ldots, \Gamma(g, h) u_{q}\right)\right\} \\
& -(g, h)^{*}\left\{\left(g^{-1}, h^{-1}\right)^{*} \omega\left(\Gamma(g, h) u_{1}, \ldots,\left[\Gamma(g, h) u, \Gamma(g, h) u_{i}\right]_{F, h}, \ldots, \Gamma(g, h) u_{q}\right)\right\}
\end{aligned}
$$

for any $\omega \in \Lambda^{q}(E, \pi, M)$ and $u_{1}, \ldots, u_{q} \in \Gamma(E, \pi, M)$, will be called the covariant Lie $(g, h)$-derivative with respect to the section $u$.

Definition 3.4. For any $a=1, \cdots, r$, we consider the real function $U^{a}$ on $E$ such that

$$
\left.U^{a}\right|_{\pi^{-1}(V)}\left(u_{x}\right)=y^{a},
$$

where the real numbers $y^{1}, \cdots, y^{r}$ are the fibre components of the point $u_{x}$ in the arbitrary vector local $(m+r)$-chart $\left(V, s_{V}\right)$.

Using the above definition, we can deduce $\dot{\partial}_{b}\left(U^{a}\right)=\delta_{b}^{a}$ and $\partial_{i}\left(U^{a}\right)=0$, where $a, b \in\{1, \cdots, r\}$ and $i \in\{1, \cdots, m\}$.

Now, let $\omega=\omega_{a} s^{a} \in \Lambda^{1}(E, \pi, M)$. Then we consider the real function $\hat{\omega}$ defined by

$$
\left.\hat{\omega}\right|_{\pi^{-1}(V)}=\left.U^{a}\left(\omega_{a} \circ \pi\right)\right|_{\pi^{-1}(V)},
$$

where $\left(V, s_{V}\right)$ is an arbitrary vector local $(m+r)$-chart.

Theorem 3.5. Let $u$ be a section of $(E, \pi, M)$. Then there exists a unique vector field $u^{c} \in \Gamma\left(T E, \tau_{E}, E\right)$, the complete $(g, h)$-lift of $u$, satisfying the following conditions:

i) $u^{c}$ is $(h \circ \pi)$-related with $\Gamma(T h \circ \rho, h \circ \eta)(\Gamma(g, h) u)$, i.e.,

$$
T(h \circ \pi)\left(u_{v_{x}}^{c}\right)=\{\Gamma(T h \circ \rho, h \circ \eta)(\Gamma(g, h) u)\}\left(h \circ \pi\left(v_{x}\right)\right),
$$

ii) $u^{c}(\hat{\omega})=\left(\widehat{g, h) \mathcal{L}_{u}} \omega, \quad \forall \omega \in \Lambda^{1}(E, \pi, M)\right.$.

Proof. At first we let that there exists $u^{c}$ such that it satisfies in (i) and (ii). Since $u^{c}$ is a vector field on $E$, then we can write it as follows:

$$
u^{c}=A^{i} \partial_{i}+B^{a} \dot{\partial}_{a}
$$

where $A^{i}, B^{a} \in \mathcal{F}(E)$. We have

$$
T(h \circ \pi)\left(\partial_{i v_{x}}\right)(f)=T \pi\left(\partial_{i v_{x}}\right)(f \circ h)=\partial_{i v_{x}}(f \circ h \circ \pi),
$$

and

$$
T(h \circ \pi)\left(\dot{\partial}_{a v_{x}}\right)(f)=T \pi\left(\dot{\partial}_{a v_{x}}\right)(f \circ h)=\dot{\partial}_{a v_{x}}(f \circ h \circ \pi)=0 .
$$

From two above equations we obtain

$$
T(h \circ \pi)\left(u_{v_{x}}^{c}\right)(f)=A^{i}\left(v_{x}\right) \partial_{i v_{x}}(f \circ h \circ \pi) .
$$


On the other hand we have

$$
\begin{aligned}
& \Gamma(T h \circ \rho, h \circ \eta)(\Gamma(g, h) u) h \circ \pi\left(v_{x}\right)(f) \\
& =\left(\left(g_{c}^{\alpha} \circ \pi\right)\left(u^{c} \circ \pi\right)\left(\rho_{\alpha}^{i} \circ h \circ \pi\right)\right)\left(v_{x}\right) \partial_{i v_{x}}(f \circ h \circ \pi) .
\end{aligned}
$$

Condition (i) gives us $A^{i}=\left(g_{c}^{\alpha} u^{c} \rho_{\alpha}^{i} \circ h\right) \circ \pi$. Therefore we have

$$
u^{c}=\left(\left(g_{c}^{\alpha} u^{c} \rho_{\alpha}^{i} \circ h\right) \circ \pi\right) \partial_{i}+B^{a} \dot{\partial}_{a} .
$$

Now, let $\omega=\omega_{b} s^{b} \in \Lambda^{1}(E, \pi, M)$. Then we get

$$
u^{c}(\hat{\omega})=U^{b}\left(\left(g_{c}^{\alpha} u^{c} \rho_{\alpha}^{i} \circ h\right) \circ \pi\right) \partial_{i}\left(\omega_{b} \circ \pi\right)+B^{b}\left(\omega_{b} \circ \pi\right) .
$$

Now, let $K_{a}^{\gamma}(u) t_{\gamma}=\left[\Gamma(g, h) u, \Gamma(g, h) s_{a}\right]_{F, h}$. Then we get

$$
\begin{aligned}
K_{a}^{\gamma}(u)= & \left(g_{c}^{\beta} u^{c}\right) \circ h^{-1} \rho_{\beta}^{j} \frac{\partial g_{a}^{\gamma}}{\partial x^{i}} \circ h^{-1}-\left(g_{a}^{\alpha} \circ h^{-1}\right) \rho_{\alpha}^{i} \frac{\partial\left(g_{c}^{\gamma} u^{c}\right)}{\partial x^{i}} \circ h^{-1} \\
& +\left(g_{c}^{\alpha} u^{c}\right) \circ h^{-1} L_{\alpha \beta}^{\gamma}\left(g_{a}^{\beta} \circ h^{-1}\right) .
\end{aligned}
$$

On the other hand we have

$$
\begin{aligned}
(g, h) \mathcal{L}_{u} \omega\left(s_{a}\right)= & (g, h)^{*}\left\{\Gamma(T h \circ \rho, h \circ \eta)(\Gamma(g, h) u)\left(g^{-1}, h^{-1}\right)^{*} \omega\left(\Gamma(g, h) s_{a}\right)\right\} \\
& -(g, h)^{*}\left\{\left(g^{-1}, h^{-1}\right)^{*} \omega\left(K_{a}^{\gamma}(u) t_{\gamma}\right)\right\} \\
= & g_{b}^{\alpha} u^{b}\left(\rho_{\alpha}^{i} \circ h\right) \frac{\partial \omega_{a}}{\partial x^{i}}-\tilde{g}_{\gamma}^{b} \omega_{b} K_{a}^{\gamma}(u) \circ h \\
= & g_{b}^{\alpha} u^{b}\left(\rho_{\alpha}^{i} \circ h\right) \frac{\partial \omega_{a}}{\partial x^{i}}-g_{c}^{\beta} u^{c}\left(\rho_{\beta}^{j} \circ h\right) \frac{\partial g_{a}^{\gamma}}{x^{i}} \tilde{g}_{\gamma}^{b} \omega_{b} \\
& -g_{a}^{\alpha}\left(\rho_{\alpha}^{i} \circ h\right) \frac{\left.\partial g_{c}^{\gamma} u^{c}\right)}{\partial x^{i}} \tilde{g}_{\gamma}^{b} \omega_{b}+g_{c}^{\alpha} u^{c}\left(L_{\alpha \beta}^{\gamma} \circ h\right) g_{a}^{\beta} \tilde{g}_{\gamma}^{b} \omega_{b} .
\end{aligned}
$$

Thus we have

$$
\begin{aligned}
\left(\widehat{g, h) \mathcal{L}_{u}} \omega=\right. & U^{a}\left((g, h) \mathcal{L}_{u} \omega\left(s_{a}\right)\right) \circ \pi \\
= & U^{a}\left(g_{b}^{\alpha} u^{b}\left(\rho_{\alpha}^{i} \circ h\right) \frac{\partial \omega_{a}}{\partial x^{i}}-\tilde{g}_{\gamma}^{b} \omega_{b} K_{a}^{\gamma}(u) \circ h\right) \circ \pi \\
= & U^{a}\left\{\left(g_{b}^{\alpha} u^{b}\left(\rho_{\alpha}^{i} \circ h\right) \frac{\partial \omega_{a}}{\partial x^{i}}-\tilde{g}_{\gamma}^{b} \omega_{b} g_{c}^{\beta} u^{c}\left(\rho_{\beta}^{j} \circ h\right) \frac{\partial g_{a}^{\gamma}}{\partial x^{i}}\right) \circ \pi\right. \\
& \left.+\left(-g_{a}^{\alpha}\left(\rho_{\alpha}^{i} \circ h\right) \frac{\partial\left(g_{c}^{\gamma} u^{c}\right)}{\partial x^{i}} \tilde{g}_{\gamma}^{b} \omega_{b}+g_{c}^{\alpha} u^{c}\left(L_{\alpha \beta}^{\gamma} \circ h\right) g_{a}^{\beta} \tilde{g}_{\gamma}^{b} \omega_{b}\right) \circ \pi\right\} .
\end{aligned}
$$

But condition (ii) gives us

$$
B^{b}\left(\omega_{b} \circ \pi\right)=-U^{a}\left(K_{a}^{\gamma}(u) \circ h \circ \pi\right)\left(\left(\tilde{g}_{\gamma}^{b} \omega_{b}\right) \circ \pi\right) .
$$

Since $\omega$ is arbitrary, then we suppose that $\omega=s^{b}$. Thus we have $\omega_{b}=1$ and $\omega_{a}=0$, for any $a \neq b$. Therefore we obtain

$$
B^{b}=-U^{a}\left(K_{a}^{\gamma}(u) \circ h \circ \pi\right)\left(\tilde{g}_{\gamma}^{b} \circ \pi\right) .
$$

So, for $u^{c}$ we can obtain the following locally expression:

$$
u^{c}=\left(g_{e}^{\alpha} u^{e} \rho_{\alpha}^{i} \circ h\right) \circ \pi \partial_{i}-U^{a}\left(K_{a}^{\gamma}(u) \circ h \circ \pi\right)\left(\tilde{g}_{\gamma}^{b} \circ \pi\right) \dot{\partial}_{b} .
$$

The above relation proves the existence and uniqueness of the complete lift.

Definition 3.6. The complete $(g, h)$-lift of a function $f \in \mathcal{F}(N)$ into $\mathcal{F}(E)$ is the function

$$
f^{c}: E \longrightarrow \mathbb{R}
$$


defined by

$$
\left.f^{c}\right|_{\pi^{-1}(V)}=\left.U^{a}\left(g_{a}^{b} \circ \pi\right)\left(\rho_{b}^{i} \circ h \circ \pi\right) \partial_{i}(f \circ h \circ \pi)\right|_{\pi^{-1}(V)},
$$

where $\left(V, s_{V}\right)$ is an arbitrary vector local $(m+r)$-chart.

Lemma 3.7. If $u$ is a section of $(E, \pi, M)$ and $f, f_{1}, f_{2} \in \mathcal{F}(N)$, then

(i) $\left(f_{1}+f_{2}\right)^{c}=f_{1}^{c}+f_{2}^{c}$,

(ii) $\left(f_{1} f_{2}\right)^{c}=f_{1}^{c} f_{2}^{\vee}+f_{1}^{\vee} f_{2}^{c}$,

(iii) $u^{\vee}\left(f^{c}\right)=\{\Gamma(T h \circ \rho, h \circ \eta)(\Gamma(g, h) u)(f)\}^{\vee}$.

Proof. We only prove (iii). Using (3.1), 3.2 and 3.4 we obtain

$$
\begin{aligned}
u^{\vee}\left(f^{c}\right) & =\left(u^{b} \circ \pi\right) \dot{\partial}_{b}\left(U^{b}\left(g_{b}^{\alpha} \circ \pi\right)\left(\rho_{\alpha}^{i} \circ h \circ \pi\right) \partial_{i}(f \circ h \circ \pi)\right) \\
& =\left(g_{b}^{\alpha} \circ \pi\right)\left(u^{b} \circ \pi\right)\left(\rho_{\alpha}^{i} \circ h \circ \pi\right) \partial_{i}(f \circ h \circ \pi) \\
& =\left\{\left(g_{b}^{\alpha} \circ h^{-1}\right)\left(u^{b} \circ h^{-1}\right) \rho_{\alpha}^{i}\left(\left(\partial_{i}(f \circ h)\right) \circ h^{-1}\right)\right\} \circ h \circ \pi \\
& =\{\Gamma(T h \circ \rho, h \circ \eta)(\Gamma(g, h) u)(f)\}^{\vee} .
\end{aligned}
$$

Lemma 3.8. Let $u$ be a section of $(E, \pi, M)$. Then

$$
u^{c}\left(f^{c}\right)=\left\{(\Gamma(T h \circ \rho, h \circ \eta)(\Gamma(g, h) u)(f)\}^{c}, \quad \forall f \in \mathcal{F}(N) .\right.
$$

Proof. Using (3.3) and (3.4) we get

$$
\begin{aligned}
& \left\{(\Gamma(T h \circ \rho, h \circ \eta)(\Gamma(g, h) u)(f)\}^{c}\right. \\
& =\left(\left(g_{e}^{\alpha} \circ h^{-1}\right)\left(u^{e} \circ h^{-1}\right) \rho_{\alpha}^{i} \partial_{i}(f \circ h) \circ h^{-1}\right)^{c} \\
& =U^{b}\left(g_{b}^{\beta} \circ \pi\right)\left(\rho_{\beta}^{j} \circ h \circ \pi\right) \partial_{j}\left(\left(g_{e}^{\alpha} \circ \pi\right)\left(u^{e} \circ \pi\right)\left(\rho_{\alpha}^{i} \circ h \circ \pi\right) \partial_{i}(f \circ h \circ \pi)\right) \\
& =U^{b}\left(g_{b}^{\beta} \circ \pi\right)\left(\rho_{\beta}^{j} \circ h \circ \pi\right)\left(\partial_{j}\left(g_{e}^{\alpha} \circ \pi\right)\right)\left(u^{e} \circ \pi\right)\left(\rho_{\alpha}^{i} \circ h \circ \pi\right) \partial_{i}(f \circ h \circ \pi) \\
& +U^{b}\left(g_{b}^{\beta} \circ \pi\right)\left(\rho_{\beta}^{j} \circ h \circ \pi\right)\left(g_{e}^{\alpha} \circ \pi\right)\left(\partial_{j}\left(u^{e} \circ \pi\right)\right)\left(\rho_{\alpha}^{i} \circ h \circ \pi\right) \partial_{i}(f \circ h \circ \pi) \\
& +U^{b}\left(g_{b}^{\beta} \circ \pi\right)\left(\rho_{\beta}^{j} \circ h \circ \pi\right)\left(g_{e}^{\alpha} \circ \pi\right)\left(u^{e} \circ \pi\right)\left(\partial_{j}\left(\rho_{\alpha}^{i} \circ h \circ \pi\right)\right) \partial_{i}(f \circ h \circ \pi) \\
& +U^{b}\left(g_{b}^{\beta} \circ \pi\right)\left(\rho_{\beta}^{j} \circ h \circ \pi\right)\left(g_{e}^{\alpha} \circ \pi\right)\left(u^{e} \circ \pi\right)\left(\rho_{\alpha}^{i} \circ h \circ \pi\right) \partial_{j} \partial_{i}(f \circ h \circ \pi) .
\end{aligned}
$$


Again, (3.3) and (3.4) give us

$$
\begin{aligned}
& u^{c}\left(f^{c}\right)=\left(g_{e}^{\alpha} \circ \pi\right)\left(u^{e} \circ \pi\right)\left(\rho_{\alpha}^{i} \circ h \circ \pi\right) \partial_{i}\left(U^{b}\left(g_{b}^{\beta} \circ \pi\right)\left(\rho_{\beta}^{j} \circ h \circ \pi\right) \partial_{j}(f \circ h \circ \pi)\right) \\
& -U^{a}\left(K_{a}^{\gamma}(u) \circ h \circ \pi\right)\left(\tilde{g}_{\gamma}^{b} \circ \pi\right) \dot{\partial}_{b}\left(U^{b}\left(g_{b}^{\beta} \circ \pi\right)\left(\rho_{\beta}^{j} \circ h \circ \pi\right) \partial_{j}(f \circ h \circ \pi)\right) \\
& =U^{b}\left(g_{e}^{\alpha} \circ \pi\right)\left(u^{e} \circ \pi\right)\left(\rho_{\alpha}^{i} \circ h \circ \pi\right)\left(\partial_{i}\left(g_{b}^{\beta} \circ \pi\right)\right)\left(\rho_{\beta}^{j} \circ h \circ \pi\right) \partial_{j}(f \circ h \circ \pi) \\
& +U^{b}\left(g_{e}^{\alpha} \circ \pi\right)\left(u^{e} \circ \pi\right)\left(\rho_{\alpha}^{i} \circ h \circ \pi\right)\left(g_{b}^{\beta} \circ \pi\right)\left(\partial_{i}\left(\rho_{\beta}^{j} \circ h \circ \pi\right)\right) \partial_{j}(f \circ h \circ \pi) \\
& +U^{b}\left(g_{e}^{\alpha} \circ \pi\right)\left(u^{e} \circ \pi\right)\left(\rho_{\alpha}^{i} \circ h \circ \pi\right)\left(g_{b}^{\beta} \circ \pi\right)\left(\rho_{\beta}^{j} \circ h \circ \pi\right) \partial_{i} \partial_{j}(f \circ h \circ \pi) \\
& -U^{a}\left(K_{a}^{\beta}(u) \circ h \circ \pi\right)\left(\rho_{\beta}^{j} \circ h \circ \pi\right) \partial_{j}(f \circ h \circ \pi) \\
& =U^{b}\left(g_{e}^{\alpha} \circ \pi\right)\left(u^{e} \circ \pi\right)\left(\rho_{\alpha}^{i} \circ h \circ \pi\right)\left(\partial_{i}\left(g_{b}^{\beta} \circ \pi\right)\right)\left(\rho_{\beta}^{j} \circ h \circ \pi\right) \partial_{j}(f \circ h \circ \pi) \\
& +U^{b}\left(g_{e}^{\alpha} \circ \pi\right)\left(u^{e} \circ \pi\right)\left(\rho_{\alpha}^{i} \circ h \circ \pi\right)\left(g_{b}^{\beta} \circ \pi\right)\left(\partial_{i}\left(\rho_{\beta}^{j} \circ h \circ \pi\right)\right) \partial_{j}(f \circ h \circ \pi) \\
& +U^{b}\left(g_{e}^{\alpha} \circ \pi\right)\left(u^{e} \circ \pi\right)\left(\rho_{\alpha}^{i} \circ h \circ \pi\right)\left(g_{b}^{\beta} \circ \pi\right)\left(\rho_{\beta}^{j} \circ h \circ \pi\right) \partial_{i} \partial_{j}(f \circ h \circ \pi) \\
& -U^{b}\left(\left(g_{c}^{\beta} u^{c}\right) \circ \pi\left(\rho_{\beta}^{j} \circ h \circ \pi\right) \partial_{j}\left(g_{b}^{\gamma} \circ \pi\right)\right)\left(\rho_{\gamma}^{k} \circ h \circ \pi\right) \partial_{k}(f \circ h \circ \pi) \\
& +U^{b}\left(g_{b}^{\alpha} \circ \pi\right) \rho_{\alpha}^{i} \circ h \circ \pi\left(\partial_{i}\left(g_{c}^{\gamma} u^{c}\right) \circ \pi\right)\left(\rho_{\gamma}^{k} \circ h \circ \pi\right) \partial_{k}(f \circ h \circ \pi) \\
& -U^{b}\left(g_{c}^{\alpha} u^{c}\right) \circ \pi\left(L_{\alpha \beta}^{\gamma} \circ h \circ \pi\right)\left(g_{b}^{\beta} \circ \pi\right)\left(\rho_{\gamma}^{k} \circ h \circ \pi\right) \partial_{k}(f \circ h \circ \pi) .
\end{aligned}
$$

Using 2.1 in the above equation, we obtain the assertion.

Definition 3.9. The complete $(g, h)$-lift $u^{C}$ of a section $u \in \Gamma(E, \pi, M)$ is the section of $\left((\rho, \eta) T E,(\rho, \eta) \tau_{E}, E\right)$ given by

$$
\begin{aligned}
& u^{C}=\left(g_{e}^{\alpha} \circ \pi\right)\left(u^{e} \circ \pi\right) T_{\alpha} \oplus\left(g_{e}^{\alpha} \circ \pi\right)\left(u^{e} \circ \pi\right)\left(\rho_{\alpha}^{i} \circ h \circ \pi\right) \partial_{i} \\
& -U^{a}\left(K_{a}^{\gamma}(u) \circ h \circ \pi\right)\left(\tilde{g}_{\gamma}^{b} \circ \pi\right)\left(0_{(h \circ \pi) * E} \oplus \dot{\partial}_{b}\right) \\
& =\left(\left(g_{e}^{\alpha} \circ \pi\right)\left(u^{e} \circ \pi\right)\right) T_{\alpha} \oplus\left(\rho_{\alpha}^{i} \circ h \circ \pi\right) \partial_{i} \\
& -U^{a}\left(K_{a}^{\gamma}(u) \circ h \circ \pi\right)\left(\tilde{g}_{\gamma}^{b} \circ \pi\right)\left(0_{(h \circ \pi) * E} \oplus \dot{\partial}_{b}\right) \\
& =\left(\left(g_{e}^{\alpha} \circ \pi\right)\left(u^{e} \circ \pi\right)\right) \tilde{\partial}_{\alpha}-U^{a}\left(K_{a}^{\gamma}(u) \circ h \circ \pi\right)\left(\tilde{g}_{\gamma}^{b} \circ \pi\right) \dot{\tilde{\partial}}_{b} .
\end{aligned}
$$

Using the above definition, we can obtain

$$
\Gamma\left(\tilde{\rho}, I d_{E}\right)\left(u^{C}\right)=u^{c} .
$$

In the particular case of Lie algebroids, $(g, \eta, h)=\left(I d_{E}, I d_{M}, I d_{M}\right)$, the complete lifts are given by the equality

$$
\begin{gathered}
u^{c}=\left\{\left(u^{a} \rho_{a}^{i}\right) \circ \pi\right\} \partial_{i}+y^{b}\left\{\left(\rho_{b}^{i} \partial_{i} u^{a}+u^{d} L_{b d}^{a}\right) \circ \pi\right\} \dot{\partial}_{a}, \\
u^{C}=\left(u^{a} \circ \pi\right) \tilde{\partial}_{a}+y^{b}\left\{\left(\rho_{b}^{i} \partial_{i} u^{a}+u^{d} L_{b d}^{a}\right) \circ \pi\right\} \dot{\tilde{\partial}}_{a},
\end{gathered}
$$

and in the classical case, $\rho=I d_{T M}$, the complete lifts are given by the equality

$$
u^{C}=\left(X^{i} \circ \pi\right) \partial_{i}+y^{j}\left(\partial_{j} X^{i} \circ \pi\right) \dot{\partial}_{i}=u^{c} .
$$

Definition 3.10. If $u=u^{a} s_{a}$ is a section of $(E, \pi, M)$, then we introduce the vertical $(g, h)$-lift of $u$ as a section of $\left((\rho, \eta) T E,(\rho, \eta) \tau_{E}, E\right)$ given by

$$
u^{V}=0_{(h \circ \pi)^{*} E} \oplus u^{\vee} .
$$


If $u=u^{a} e_{a} \in \Gamma(E, \pi, M)$, then in the locally expressions we get

$$
u^{V}=0_{(h \circ \pi)^{*} E} \oplus\left(u^{a} \circ \pi\right) \dot{\partial}_{a}=\left(u^{a} \circ \pi\right)\left(0_{(h \circ \pi)^{*} E} \oplus \dot{\partial}_{a}\right)=\left(u^{a} \circ \pi\right) \dot{\tilde{\partial}}_{a} .
$$

In particular, we have $s_{a}^{V}=\dot{\tilde{\partial}}_{a}$.

Remark 3.11. Using the almost tangent $(g, h)$-structure $\mathcal{J}_{(g, h)}$ given by

$$
\mathcal{J}_{(g, h)}\left(Z^{\alpha} \tilde{\partial}_{\alpha}+Y^{b} \dot{\tilde{\partial}}_{b}\right)=\left(\tilde{g}_{\alpha}^{b} \circ \pi\right) Z^{\alpha} \dot{\tilde{\partial}}_{b}
$$

it results that $\mathcal{J}_{(g, h)}\left(u^{C}\right)=u^{V}$.

Theorem 3.12. The Lie brackets of vertical and complete $(g, h)$-lifts satisfy the following equalities:

$$
\begin{aligned}
\text { i) }\left[u^{V}, v^{V}\right]_{(\rho, \eta) T E} & =0 \\
\text { ii) }\left[u^{V}, v^{C}\right]_{(\rho, \eta) T E} & =\left\{\Gamma\left(g^{-1}, h^{-1}\right)[\Gamma(g, h) u, \Gamma(g, h) v]_{F, h}\right\}^{V}, \\
\text { iii) }\left[u^{C}, v^{C}\right]_{(\rho, \eta) T E} & =\left\{\Gamma\left(g^{-1}, h^{-1}\right)[\Gamma(g, h) u, \Gamma(g, h) v]_{F, h}\right\}^{C} .
\end{aligned}
$$

Proof. Direct calculation gives us

$$
\begin{aligned}
{\left[u^{V}, v^{C}\right]_{(\rho, \eta) T E}=} & -\left\{( ( u ^ { a } \tilde { g } _ { \gamma } ^ { b } ) \circ \pi ) \left(\left(\left(g_{c}^{\beta} v^{c}\left(\rho_{\beta}^{j} \circ h\right)\right) \circ \pi\right) \partial_{j}\left(g_{a}^{\gamma} \circ \pi\right)\right.\right. \\
& \left.-\left(\left(g_{a}^{\alpha}\left(\rho_{\alpha}^{i} \circ h\right)\right) \circ \pi\right) \partial_{i}\left(\left(g_{c}^{\gamma} v^{c}\right) \circ \pi\right)+\left(\left(g_{c}^{\alpha} v^{c} g_{a}^{\beta}\left(L_{\alpha \beta}^{\gamma} \circ h\right)\right) \circ \pi\right)\right) \\
& \left.+\left(\left(g_{c}^{\alpha} v^{c}\left(\rho_{\alpha}^{i} \circ h\right)\right) \circ \pi\right) \partial_{i}\left(u^{b} \circ \pi\right)\right\} \dot{\tilde{\partial}}_{b} .
\end{aligned}
$$

On the other hand, we have

$$
\begin{aligned}
{[\Gamma} & (g, h)(u), \Gamma(g, h)(v)]_{F, h}=\left[\left(\left(g_{a}^{\alpha} u^{a}\right) \circ h^{-1}\right) t_{\alpha},\left(\left(g_{b}^{\beta} v^{b}\right) \circ h^{-1}\right) t_{\beta}\right]_{F, h} \\
= & \left(\left(g_{a}^{\alpha} u^{a}\right) \circ h^{-1}\right) \Gamma(T h \circ \rho, h \circ \eta)\left(t_{\alpha}\right)\left(\left(g_{b}^{\beta} v^{b}\right) \circ h^{-1}\right) t_{\beta} \\
& -\left(\left(g_{b}^{\beta} v^{b}\right) \circ h^{-1}\right) \Gamma(T h \circ \rho, h \circ \eta)\left(t_{\beta}\right)\left(\left(g_{a}^{\alpha} u^{a}\right) \circ h^{-1}\right) t_{\alpha} \\
& +\left(\left(g_{a}^{\alpha} u^{a} g_{b}^{\beta} v^{b}\right) \circ h^{-1}\right) L_{\alpha \beta}^{\gamma} t_{\gamma} \\
= & \left\{\left(\left(g_{a}^{\alpha} u^{a}\right) \circ h^{-1}\right) \rho_{\alpha}^{i} \frac{\partial\left(g_{b}^{\gamma} v^{b}\right)}{\partial x^{i}} \circ h^{-1}-\left(\left(g_{b}^{\beta} v^{b}\right) \circ h^{-1}\right) \rho_{\beta}^{i} \frac{\partial\left(g_{a}^{\gamma} u^{a}\right)}{\partial x^{i}} \circ h^{-1}\right. \\
& \left.+\left(\left(g_{a}^{\alpha} u^{a} g_{b}^{\beta} v^{b}\right) \circ h^{-1}\right) L_{\alpha \beta}^{\gamma}\right\} t_{\gamma} .
\end{aligned}
$$

The above equation gives us

$$
\begin{aligned}
& \Gamma\left(g^{-1}, h^{-1}\right)\left([\Gamma(g, h)(u), \Gamma(g, h)(v)]_{F, h}=\tilde{g}_{\gamma}^{d}\left\{g_{a}^{\alpha} u^{a}\left(\rho_{\alpha}^{i} \circ h\right) \frac{\partial\left(g_{b}^{\gamma} v^{b}\right)}{\partial x^{i}}\right.\right. \\
& \left.-g_{b}^{\beta} v^{b}\left(\rho_{\beta}^{i} \circ h\right) \frac{\partial\left(g_{a}^{\gamma} u^{a}\right)}{\partial x^{i}}+g_{a}^{\alpha} u^{a} g_{b}^{\beta} v^{b}\left(L_{\alpha \beta}^{\gamma} \circ h\right)\right\} s_{d} .
\end{aligned}
$$


Thus

$$
\begin{aligned}
& \left(\Gamma\left(g^{-1}, h^{-1}\right)[\Gamma(g, h)(u), \Gamma(g, h)(v)]_{F, h}\right)^{V}=\left(\tilde{g}_{\gamma}^{d} \circ \pi\right)\left\{\left(\left(v^{b} g_{a}^{\alpha} u^{a}\left(\rho_{\alpha}^{i} \circ h\right)\right) \circ \pi\right) \partial_{i}\left(g_{b}^{\gamma} \circ \pi\right)\right. \\
& \quad+\left(\left(g_{b}^{\gamma} g_{a}^{\alpha} u^{a}\left(\rho_{\alpha}^{i} \circ h\right)\right) \circ \pi\right) \partial_{i}\left(v^{b} \circ \pi\right)-\left(\left(u^{a} g_{b}^{\beta} v^{b}\left(\rho_{\beta}^{i} \circ h\right)\right) \circ \pi\right) \partial_{i}\left(g_{a}^{\gamma} \circ \pi\right) \\
& \left.\quad-\left(\left(g_{a}^{\gamma} g_{b}^{\beta} v^{b}\left(\rho_{\beta}^{i} \circ h\right)\right) \circ \pi\right) \partial_{i}\left(u^{a} \circ \pi\right)+\left(g_{a}^{\alpha} u^{a} g_{b}^{\beta} v^{b}\left(L_{\alpha \beta}^{\gamma} \circ h\right)\right) \circ \pi\right\} \dot{\tilde{\partial}}_{d} .
\end{aligned}
$$

From (3.7) and (3.8) we get (ii). Now we prove (iii). we have

$$
\left\{\Gamma\left(g^{-1}, h^{-1}\right)[\Gamma(g, h) u, \Gamma(g, h) v]_{F, h}\right\}^{C}=\mathcal{A}^{\gamma} \tilde{\partial}_{\gamma}+\mathcal{B}^{r} \dot{\tilde{\partial}}_{r}
$$

where

$$
\begin{aligned}
\mathcal{A}^{\gamma}= & \left(\left(g_{e}^{\alpha} u^{e}\left(\rho_{\alpha}^{i} \circ h\right)\right) \circ \pi\right) \partial_{i}\left(\left(g_{c}^{\lambda} v^{c}\right) \circ \pi\right)-\left(\left(g_{c}^{\beta} v^{c}\left(\rho_{\beta}^{i} \circ h\right)\right) \circ \pi\right) \partial_{i}\left(\left(g_{e}^{\gamma} u^{e}\right) \circ \pi\right) \\
& +\left(\left(g_{e}^{\alpha} u^{e} g_{c}^{\beta} v^{c}\left(L_{\alpha \beta}^{\gamma} \circ h\right) \circ \pi\right),\right.
\end{aligned}
$$

and

$$
\begin{aligned}
\mathcal{B}^{r}= & -U^{d}\left(\tilde{g}_{\gamma}^{r} \circ \pi\right)\left\{\left(\left(g_{a}^{\alpha} u^{a}\left(\left(\rho_{\alpha}^{i} \rho_{\lambda}^{j}\right) \circ h\right)\right) \circ \pi\right) \partial_{i}\left(\left(g_{b}^{\lambda} v^{b}\right) \circ \pi\right) \partial_{j}\left(g_{d}^{\gamma} \circ \pi\right)\right. \\
& -\left(\left(g_{b}^{\beta} v^{b}\left(\left(\rho_{\beta}^{i} \rho_{\lambda}^{j}\right) \circ h\right)\right) \circ \pi\right) \partial_{i}\left(\left(g_{a}^{\lambda} u^{a}\right) \circ \pi\right) \partial_{j}\left(g_{d}^{\gamma} \circ \pi\right) \\
& +\left(\left(g_{a}^{\alpha} u^{a} g_{b}^{\beta} v^{b}\left(\left(L_{\alpha \beta}^{\lambda} \rho_{\lambda}^{j}\right) \circ h\right)\right) \circ \pi\right) \partial_{j}\left(g_{d}^{\gamma} \circ \pi\right) \\
& -\left(\left(g_{d}^{\lambda}\left(\left(\rho_{\lambda}^{j} \rho_{\alpha}^{i}\right) \circ h\right)\right) \circ \pi\right) \partial_{j}\left(\left(g_{a}^{\alpha} u^{a}\right) \circ \pi\right) \partial_{i}\left(\left(g_{b}^{\gamma} v^{b}\right) \circ \pi\right) \\
& -\left(\left(g_{d}^{\lambda} g_{a}^{\alpha} u^{a}\left(\rho_{\lambda}^{j} \circ h\right)\right) \circ \pi\right) \partial_{j}\left(\rho_{\alpha}^{i} \circ h \circ \pi\right) \partial_{i}\left(\left(g_{b}^{\gamma} v^{b}\right) \circ \pi\right) \\
& -\left(\left(g_{d}^{\lambda} g_{a}^{\alpha} u^{a}\left(\left(\rho_{\alpha}^{i} \rho_{\lambda}^{j}\right) \circ h\right)\right) \circ \pi\right) \partial_{j} \partial_{i}\left(\left(g_{b}^{\gamma} v^{b}\right) \circ \pi\right) \\
& +\left(\left(g_{d}^{\lambda}\left(\left(\rho_{\lambda}^{j} \rho_{\beta}^{i}\right) \circ h\right)\right) \circ \pi\right) \partial_{j}\left(\left(g_{b}^{\beta} v^{b}\right) \circ \pi\right) \partial_{i}\left(\left(g_{a}^{\gamma} u^{a}\right) \circ \pi\right) \\
& +\left(\left(g_{d}^{\lambda} g_{b}^{\beta} v^{b}\left(\rho_{\lambda}^{j} \circ h\right)\right) \circ \pi\right) \partial_{j}\left(\rho_{\beta}^{i} \circ h \circ \pi\right) \partial_{i}\left(\left(g_{a}^{\gamma} u^{a}\right) \circ \pi\right) \\
& +\left(\left(g_{d}^{\lambda} g_{b}^{\beta} v^{b}\left(\left(\rho_{\beta}^{i} \rho_{\lambda}^{j}\right) \circ h\right)\right) \circ \pi\right) \partial_{j} \partial_{i}\left(\left(g_{a}^{\gamma} u^{a}\right) \circ \pi\right) \\
& -\left(\left(g_{d}^{\lambda} g_{b}^{\beta} v^{b}\left(\left(L_{\alpha \beta}^{\gamma} \rho_{\lambda}^{j}\right) \circ h\right)\right) \circ \pi\right) \partial_{j}\left(\left(g_{a}^{\alpha} u^{a}\right) \circ \pi\right) \\
& -\left(\left(g_{d}^{\lambda} g_{a}^{\alpha} u^{a}\left(\left(L_{\alpha \beta}^{\gamma} \rho_{\lambda}^{j}\right) \circ h\right)\right) \circ \pi\right) \partial_{j}\left(\left(g_{b}^{\beta} v^{b}\right) \circ \pi\right) \\
& -\left(\left(g_{d}^{\lambda} g_{a}^{\alpha} u^{a} g_{b}^{\beta} v^{b}\left(\rho_{\lambda}^{j} \circ h\right)\right) \circ \pi\right) \partial_{j}\left(L_{\alpha \beta}^{\gamma} \circ h \circ \pi\right) \\
& +\left(\left(g_{d}^{\mu} g_{a}^{\alpha} u^{a}\left(\left(L_{\lambda \mu}^{\gamma} \rho_{\alpha}^{i}\right) \circ h\right)\right) \circ \pi\right) \partial_{i}\left(\left(g_{b}^{\lambda} v^{b}\right) \circ \pi\right) \\
& -\left(\left(g_{d}^{\mu} g_{b}^{\beta} v^{b}\left(\left(L_{\lambda \mu}^{\gamma} \rho_{\beta}^{i}\right) \circ h\right)\right) \circ \pi\right) \partial_{i}\left(\left(g_{a}^{\lambda} u^{a}\right) \circ \pi\right) \\
& \left.+\left(\left(g_{d}^{\mu} g_{a}^{\alpha} u^{a} g_{b}^{\beta} v^{b}\left(\left(L_{\lambda \mu}^{\gamma} L_{\alpha \beta}^{\lambda}\right) \circ h\right)\right) \circ \pi\right)\right\} .
\end{aligned}
$$

Using 3.5 and direct calculation we get

$$
\left[u^{C}, v^{C}\right]_{(\rho, \eta) T E}=\mathcal{A}^{\gamma} \tilde{\partial}_{\gamma}+\left(\mathcal{B}^{r}+\mathcal{C}^{r}\right) \dot{\tilde{\partial}}_{r},
$$


where

$$
\begin{aligned}
\mathcal{C}^{r}= & -U^{d}\left(\tilde{g}_{\gamma}^{r} \circ \pi\right)\left\{-\left(\left(\tilde{g}_{\alpha}^{a} g_{c}^{\lambda} u^{c} g_{e}^{\sigma} v^{e}\left(\left(\rho_{\sigma}^{i} \rho_{\lambda}^{j}\right) \circ h\right)\right) \circ \pi\right) \partial_{j}\left(g_{d}^{\alpha} \circ \pi\right) \partial_{i}\left(g_{a}^{\gamma} \circ \pi\right)\right. \\
& +\left(\left(\tilde{g}_{\beta}^{b} g_{c}^{\lambda} v^{c} g_{e}^{\sigma} u^{e}\left(\left(\rho_{\sigma}^{i} \rho_{\lambda}^{j}\right) \circ h\right)\right) \circ \pi\right) \partial_{j}\left(g_{d}^{\beta} \circ \pi\right) \partial_{i}\left(g_{b}^{\gamma} \circ \pi\right) \\
& +\left(\left(\tilde{g}_{\alpha}^{a} g_{d}^{\lambda} g_{e}^{\sigma} v^{e}\left(\left(\rho_{\sigma}^{i} \rho_{\lambda}^{j}\right) \circ h\right)\right) \circ \pi\right) \partial_{j}\left(\left(g_{c}^{\alpha} u^{c}\right) \circ \pi\right) \partial_{i}\left(g_{a}^{\gamma} \circ \pi\right) \\
& -\left(\left(\tilde{g}_{\beta}^{b} g_{d}^{\lambda} g_{e}^{\sigma} u^{e}\left(\left(\rho_{\sigma}^{i} \rho_{\lambda}^{j}\right) \circ h\right)\right) \circ \pi\right) \partial_{j}\left(\left(g_{c}^{\beta} v^{c}\right) \circ \pi\right) \partial_{i}\left(g_{b}^{\gamma} \circ \pi\right) \\
& -\left(\left(\tilde{g}_{\alpha}^{a} g_{c}^{\lambda} u^{c} g_{e}^{\sigma} v^{e}\left(\left(L_{\lambda \mu}^{\alpha} \rho_{\sigma}^{i}\right) \circ h\right)\right) \circ \pi\right) \partial_{i}\left(g_{a}^{\gamma} \circ \pi\right) \\
& \left.+\left(\left(\tilde{g}_{\beta}^{b} g_{c}^{\lambda} v^{c} g_{e}^{\sigma} u^{e}\left(\left(L_{\lambda \mu}^{\beta} \rho_{\sigma}^{i}\right) \circ h\right)\right) \circ \pi\right) \partial_{i}\left(g_{b}^{\gamma} \circ \pi\right)\right\} \\
& -U^{d}\left(\left(g_{e}^{\alpha} u^{e} g_{c}^{\lambda} v^{c}\left(\left(\rho_{\alpha}^{i} \rho_{\lambda}^{j}\right) \circ h\right)\right) \circ \pi\right) \partial_{i}\left(\tilde{g}_{\gamma}^{r} \circ \pi\right) \partial_{j}\left(g_{d}^{\gamma} \circ \pi\right) \\
& +U^{d}\left(\left(g_{e}^{\alpha} u^{e} g_{d}^{\lambda}\left(\left(\rho_{\alpha}^{i} \rho_{\lambda}^{j}\right) \circ h\right)\right) \circ \pi\right) \partial_{i}\left(\tilde{g}_{\gamma}^{r} \circ \pi\right) \partial_{j}\left(\left(g_{c}^{\gamma} v^{c}\right) \circ \pi\right) \\
& -U^{d}\left(\left(g_{e}^{\alpha} u^{e} g_{c}^{\lambda} v^{c} g_{d}^{\mu} \partial_{i}\left(\tilde{g}_{\gamma}^{r} \circ \pi\right)\left(\left(L_{\lambda \mu}^{\gamma} \rho_{\alpha}^{i}\right) \circ h\right)\right) \circ \pi\right) \\
& +U^{d}\left(\left(g_{e}^{\beta} v^{e} g_{c}^{\lambda} u^{c}\left(\left(\rho_{\beta}^{i} \rho_{\lambda}^{j}\right) \circ h\right)\right) \circ \pi\right) \partial_{i}\left(\tilde{g}_{\gamma}^{r} \circ \pi\right) \partial_{j}\left(g_{d}^{\gamma} \circ \pi\right) \\
& -U^{d}\left(\left(g_{e}^{\beta} v^{e} g_{d}^{\lambda}\left(\left(\rho_{\beta}^{i} \rho_{\lambda}^{j}\right) \circ h\right)\right) \circ \pi\right) \partial_{i}\left(\tilde{g}_{\gamma}^{r} \circ \pi\right) \partial_{j}\left(\left(g_{c}^{\gamma} u^{c}\right) \circ \pi\right) \\
& +U^{d}\left(\left(g_{e}^{\beta} v^{e} g_{c}^{\lambda} u^{c} g_{d}^{\mu} \partial_{i}\left(\tilde{g}_{\gamma}^{r} \circ \pi\right)\left(\left(L_{\lambda \mu}^{\gamma} \rho_{\beta}^{i}\right) \circ h\right)\right) \circ \pi\right) .
\end{aligned}
$$

On the other hand, we have

$$
g_{d}^{\gamma}=\tilde{g}_{\alpha}^{a} g_{a}^{\gamma} g_{d}^{\alpha}
$$

Derivative of the above expression with respect to $j$, we get

$$
\partial_{j}\left(g_{d}^{\gamma}\right)=-\partial_{j}\left(\tilde{g}_{\alpha}^{a}\right) g_{a}^{\gamma} g_{d}^{\alpha} .
$$

Using the above equation, we obtain

$$
\begin{aligned}
- & U^{d}\left(\left(g_{e}^{\alpha} u^{e} g_{c}^{\lambda} v^{c}\left(\left(\rho_{\alpha}^{i} \rho_{\lambda}^{j}\right) \circ h\right)\right) \circ \pi\right) \partial_{i}\left(\tilde{g}_{\gamma}^{r} \circ \pi\right) \partial_{j}\left(g_{d}^{\gamma} \circ \pi\right) \\
& =U^{d}\left(\left(g_{e}^{\alpha} u^{e} g_{c}^{\lambda} v^{c}\left(\left(\rho_{\alpha}^{i} \rho_{\lambda}^{j}\right) \circ h\right)\right) \circ \pi\right) \partial_{i}\left(\tilde{g}_{\gamma}^{r} \circ \pi\right) \partial_{j}\left(\tilde{g}_{\alpha}^{a} \circ \pi\right)\left(g_{a}^{\gamma} \circ \pi\right)\left(g_{d}^{\alpha} \circ \pi\right) \\
& =-U^{d}\left(\left(g_{e}^{\alpha} u^{e} g_{c}^{\lambda} v^{c}\left(\left(\rho_{\alpha}^{i} \rho_{\lambda}^{j}\right) \circ h\right)\right) \circ \pi\right) \partial_{i}\left(\tilde{g}_{\gamma}^{r} \circ \pi\right) \tilde{g}_{\alpha}^{a} \partial_{j}\left(g_{a}^{\gamma} \circ \pi\right)\left(g_{d}^{\alpha} \circ \pi\right) \\
& =U^{d}\left(\left(g_{e}^{\alpha} u^{e} g_{c}^{\lambda} v^{c}\left(\left(\rho_{\alpha}^{i} \rho_{\lambda}^{j}\right) \circ h\right)\right) \circ \pi\right)\left(\tilde{g}_{\gamma}^{r} \circ \pi\right) \partial_{i}\left(\tilde{g}_{\alpha}^{a} \circ \pi\right) \partial_{j}\left(g_{a}^{\gamma} \circ \pi\right)\left(g_{d}^{\alpha} \circ \pi\right) \\
& =U^{d}\left(\left(g_{e}^{\alpha} u^{e} g_{c}^{\lambda} v^{c}\left(\left(\rho_{\alpha}^{i} \rho_{\lambda}^{j}\right) \circ h\right)\right) \circ \pi\right)\left(\tilde{g}_{\gamma}^{r} \circ \pi\right)\left(\tilde{g}_{\alpha}^{a} \circ \pi\right) \partial_{j}\left(g_{a}^{\gamma} \circ \pi\right) \partial_{i}\left(g_{d}^{\alpha} \circ \pi\right) .
\end{aligned}
$$

Similarly, we have

$$
\begin{aligned}
& U^{d}\left(\left(g_{e}^{\alpha} u^{e} g_{d}^{\lambda}\left(\left(\rho_{\alpha}^{i} \rho_{\lambda}^{j}\right) \circ h\right)\right) \circ \pi\right) \partial_{i}\left(\tilde{g}_{\gamma}^{r} \circ \pi\right) \partial_{j}\left(\left(g_{c}^{\gamma} v^{c}\right) \circ \pi\right) \\
& =-U^{d}\left(\tilde{g}_{\gamma}^{r} \circ \pi\right)\left(\left(\tilde{g}_{\beta}^{b} g_{d}^{\lambda} g_{e}^{\sigma} u^{e}\left(\left(\rho_{\sigma}^{i} \rho_{\lambda}^{j}\right) \circ h\right)\right) \circ \pi\right) \partial_{j}\left(\left(g_{c}^{\beta} v^{c}\right) \circ \pi\right) \partial_{i}\left(g_{b}^{\gamma} \circ \pi\right) \\
& U^{d}\left(\left(g_{e}^{\alpha} u^{e} g_{c}^{\lambda} v^{c} g_{d}^{\mu} \partial_{i}\left(\tilde{g}_{\gamma}^{r} \circ \pi\right)\left(\left(L_{\lambda \mu}^{\gamma} \rho_{\alpha}^{i}\right) \circ h\right)\right) \circ \pi\right) \\
& =-U^{d}\left(\tilde{g}_{\gamma}^{r} \circ \pi\right)\left(\left(\tilde{g}_{\beta}^{b} g_{c}^{\lambda} v^{c} g_{e}^{\sigma} u^{e}\left(\left(L_{\lambda \mu}^{\beta} \rho_{\sigma}^{i}\right) \circ h\right)\right) \circ \pi\right) \partial_{i}\left(g_{b}^{\gamma} \circ \pi\right),
\end{aligned}
$$




$$
\begin{gathered}
U^{d}\left(\tilde{g}_{\gamma}^{r} \circ \pi\right)\left(\left(\tilde{g}_{\beta}^{b} g_{c}^{\lambda} v^{c} g_{e}^{\sigma} u^{e}\left(\left(\rho_{\sigma}^{i} \rho_{\lambda}^{j}\right) \circ h\right)\right) \circ \pi\right) \partial_{j}\left(g_{d}^{\beta} \circ \pi\right) \partial_{i}\left(g_{b}^{\gamma} \circ \pi\right) \\
=-U^{d}\left(\left(g_{e}^{\beta} v^{e} g_{c}^{\lambda} u^{c}\left(\left(\rho_{\beta}^{i} \rho_{\lambda}^{j}\right) \circ h\right)\right) \circ \pi\right) \partial_{i}\left(\tilde{g}_{\gamma}^{r} \circ \pi\right) \partial_{j}\left(g_{d}^{\gamma} \circ \pi\right), \\
U^{d}\left(\left(g_{e}^{\beta} v^{e} g_{d}^{\lambda}\left(\left(\rho_{\beta}^{i} \rho_{\lambda}^{j}\right) \circ h\right)\right) \circ \pi\right) \partial_{i}\left(\tilde{g}_{\gamma}^{r} \circ \pi\right) \partial_{j}\left(\left(g_{c}^{\gamma} u^{c}\right) \circ \pi\right) \\
=-U^{d}\left(\tilde{g}_{\gamma}^{r} \circ \pi\right)\left(\left(\tilde{g}_{\alpha}^{a} g_{d}^{\lambda} g_{e}^{\sigma} v^{e}\left(\left(\rho_{\sigma}^{i} \rho_{\lambda}^{j}\right) \circ h\right)\right) \circ \pi\right) \partial_{j}\left(\left(g_{c}^{\alpha} u^{c}\right) \circ \pi\right) \partial_{i}\left(g_{a}^{\gamma} \circ \pi\right),
\end{gathered}
$$

and

$$
\begin{aligned}
& U^{d}\left(\left(g_{e}^{\beta} v^{e} g_{c}^{\lambda} u^{c} g_{d}^{\mu} \partial_{i}\left(\tilde{g}_{\gamma}^{r} \circ \pi\right)\left(\left(L_{\lambda \mu}^{\gamma} \rho_{\beta}^{i}\right) \circ h\right)\right) \circ \pi\right) \\
& \quad=-U^{d}\left(\tilde{g}_{\gamma}^{r} \circ \pi\right)\left(\left(\tilde{g}_{\alpha}^{a} g_{c}^{\lambda} u^{c} g_{e}^{\sigma} v^{e}\left(\left(L_{\lambda \mu}^{\alpha} \rho_{\sigma}^{i}\right) \circ h\right)\right) \circ \pi\right) \partial_{i}\left(g_{a}^{\gamma} \circ \pi\right) .
\end{aligned}
$$

Setting (3.13)-(3.17) in (3.11) we deduce that $C^{r}=0$. This equation together with (3.9) and (3.10) give us (iii).

4. Vertical And COMplete $(\stackrel{*}{g}, h)$-Lifts of Sections of A DUAL Vector BUNDLE

In this section, we consider the diagram 2.3$)$, where $\left((F, \nu, N),[,]_{F, h},(\rho, \eta)\right)$ is a generalized Lie algebroid. We admit that $(\stackrel{*}{g}, h)$ is a vector bundles morphism locally invertible from $(\stackrel{*}{E}, \stackrel{*}{\pi}, M)$ to $(F, \nu, N)$ with components

$$
g^{\alpha b}, \quad \alpha \in\{1, \cdots, n\}, \quad b \in\{1, \cdots, r\} .
$$

So, for any local $(m+r)$-chart $\left(V, t_{V}\right)$ of $(\stackrel{*}{E}, \stackrel{*}{\pi}, M)$, there exists the real functions

$$
\tilde{g}_{b \alpha}: V \stackrel{\tilde{g}_{b \alpha}}{\longrightarrow} \mathbb{R}, \quad \alpha \in\{1, \cdots, n\}, \quad b \in\{1, \cdots, r\},
$$

such that

$$
\tilde{g}_{b \alpha}(\varkappa) \cdot g^{\alpha a}(\varkappa)=\delta_{b}^{a}, \quad g^{\alpha a}(\varkappa) \tilde{g}_{a \beta}(\varkappa)=\delta_{\beta}^{\alpha},
$$

for any $\varkappa \in V$. So, we can discuss about vector bundles morphism $\left(g^{*-1}, h^{-1}\right)$ from $(F, \nu, N)$ to $(\stackrel{*}{E}, \stackrel{*}{\pi}, M)$ with components $\tilde{g}_{b \alpha}, \alpha \in\{1, \cdots, n\}, b \in\{1, \cdots, r\}$.

Definition 4.1. If $f \in \mathcal{F}(N)$ (respectively $f \in \mathcal{F}(M)$ ), then the real function $f^{\vee}=f \circ h \circ \stackrel{*}{\pi}$ (respectively $f^{\vee}=f \circ \stackrel{*}{\pi}$ ) is called the vertical lift of the function $f$.

Since

$$
\left(\Gamma(T h \circ \rho, h \circ \eta) \Gamma(\stackrel{*}{g}, h)\left(u_{a} s^{a}\right)\right)(f)=\left(g^{\alpha b} u_{b}\right) \circ h^{-1} \rho_{\alpha}^{i} \frac{\partial(f \circ h)}{\partial x^{i}} \circ h^{-1},
$$

then using the above definition we obtain

$$
\left(\Gamma(T h \circ \rho, h \circ \eta) \Gamma(\stackrel{*}{g}, h)\left(u_{a} s^{a}\right)(f)\right)^{v}=\left(\left(g^{\alpha b} u_{b} \rho_{\alpha}^{i} \circ h\right) \circ \stackrel{*}{\pi}\right) \stackrel{*}{\partial}_{i}(f \circ h \circ \stackrel{*}{\pi}) .
$$


Definition 4.2. If $\stackrel{*}{u}=u_{a} s^{a}$ is a section of $(\stackrel{*}{E}, \stackrel{*}{\pi}, M)$, then we introduce the vertical lift of $\stackrel{*}{u}$ as a section of $\left(T \stackrel{*}{E}, \tau_{E}^{*}, \stackrel{*}{E}\right)$ by,

$$
\stackrel{*}{u}^{\vee}=\left(u_{a} \circ \pi\right) \dot{\partial}^{a} .
$$

If $\left\{s^{a}\right\}$ be a basis of sections of $\Gamma(\stackrel{*}{E}, \stackrel{*}{\pi}, M)$, then using the above equation we have

$$
\left(s^{a}\right)^{\vee}=\dot{\partial}^{a} .
$$

Using the locally expression of $\stackrel{* V}{u}^{\vee}$ we can deduce

Lemma 4.3. If $\stackrel{*}{\sim}$ and $\stackrel{*}{v}$ are sections of $(\stackrel{*}{E}, \stackrel{*}{\pi}, M)$ and $f \in \mathcal{F}(M)$, then

$$
(\stackrel{*}{u}+\stackrel{*}{v})^{\vee}=u^{* \vee}+*^{\vee}, \quad(f u)^{\vee}=f^{\vee} u^{*}, \quad \stackrel{* \vee}{u}\left(f^{\vee}\right)=0 .
$$

Definition 4.4. For any $\stackrel{*}{u} \in \Gamma(\stackrel{*}{E}, \stackrel{*}{\pi}, M)$, the $\mathcal{F}(M)$-multilinear application

defined by

$$
\Lambda(\stackrel{*}{E}, \stackrel{*}{\pi}, M) \stackrel{(\stackrel{*}{g}, h) \mathcal{L}_{*}^{*}}{\longrightarrow} \Lambda(\stackrel{*}{E}, \stackrel{*}{\pi}, M)
$$

$$
(\stackrel{*}{g}, h) \mathcal{L}_{u}^{*}(f)=(\stackrel{*}{g}, h)^{*}\left\{\Gamma(T h \circ \rho, h \circ \eta)[\Gamma(\stackrel{*}{g}, h) \stackrel{*}{u}]\left(\stackrel{*}{g}^{-1}, h^{-1}\right)^{*} f\right\}, \quad \forall f \in \mathcal{F}(M),
$$

and

$$
\begin{aligned}
& \left.(\stackrel{*}{g}, h) \mathcal{L}_{u_{u}^{*}}^{*}\left(\stackrel{*}{u}_{1}, \cdots, \stackrel{*}{u}\right)=(\stackrel{*}{g}, h)^{*}\left\{\mathcal{L}_{\Gamma(g, h)} \stackrel{*}{u}^{*}{ }^{-1}, h^{-1}\right){ }^{*} \stackrel{*}{\omega}\left(\Gamma(\stackrel{*}{g}, h) \stackrel{*}{u}_{1}, \cdots, \Gamma(\stackrel{*}{g}, h) \stackrel{*}{u_{q}}\right)\right\} \\
& =(\stackrel{*}{g}, h) *\left\{\Gamma(T h \circ \rho, h \circ \eta)[\Gamma(\stackrel{*}{g}, h) \stackrel{*}{u}]\left(\stackrel{*}{g}^{-1}, h^{-1}\right) * \stackrel{*}{\omega}\left(\Gamma(\stackrel{*}{g}, h) \stackrel{*}{u}_{1}, \cdots, \Gamma(\stackrel{*}{g}, h) \stackrel{*}{u_{q}}\right)\right\} \\
& -(\stackrel{*}{g}, h)^{*}\left\{\left(\stackrel{*}{g}^{-1}, h^{-1}\right){ }^{*} \stackrel{*}{\omega}\left(\Gamma(\stackrel{*}{g}, h) \stackrel{*}{u}_{1}, \cdots,\left[\Gamma(\stackrel{*}{g}, h) \stackrel{*}{u}, \Gamma(\stackrel{*}{g}, h) \stackrel{*}{u_{i}}\right]_{F, h}, \cdots, \Gamma(\stackrel{*}{g}, h) \stackrel{*}{u}_{q}\right)\right\},
\end{aligned}
$$

for any $\omega \in \Lambda^{q}(\stackrel{*}{E}, \stackrel{*}{\pi}, M)$ and $\stackrel{*}{u}_{1}, \ldots, \stackrel{*}{u}_{q} \in \Gamma(\stackrel{*}{E}, \stackrel{*}{\pi}, M)$, will be called the covariant Lie $(g, h)$-derivative with respect to the section $\stackrel{*}{u}$.

Definition 4.5. For any $a=1, \cdots, r$, we consider the real function $U_{a}$ on $\stackrel{*}{E}$ such that

$$
\left.U_{a}\right|_{\pi^{-1}(V)}\left({ }_{u}^{*}\right)=p_{a},
$$

where the real numbers $p_{1}, \cdots, p_{r}$ are the fibre components of the point $\stackrel{*}{u}_{x}$ in the arbitrary vector local $(m+r)$-chart $\left(V, s_{V}\right)$.

Using the above definition we have $\dot{\partial}^{b}\left(U_{a}\right)=\delta_{a}^{b}$ and $\partial_{i}\left(U_{a}\right)=0$, where $\alpha \in$ $\{1, \cdots, n\}, b \in\{1, \cdots, r\}$.

Definition 4.6. If $\left.\stackrel{*}{\omega}=\omega^{a} s_{a} \in \Lambda^{1} \stackrel{*}{E}, \stackrel{*}{\pi}, M\right)$, then we consider the real function $\stackrel{\widehat{*}}{\omega}$ defined by

$$
\left.\stackrel{\widehat{*}}{\omega}\right|_{\pi^{-1}(V)}=\left.U_{a}\left(\omega^{a} \circ \stackrel{*}{\pi}\right)\right|_{\pi^{-1}(V)},
$$


where $\left(V, s_{V}\right)$ is an arbitrary vector local $(m+r)$-chart.

Theorem 4.7. Let $\stackrel{*}{u}$ be a section of $(\stackrel{*}{E}, \stackrel{*}{\pi}, M)$. Then there exists a unique vector field $\stackrel{*^{c}}{u} \in \Gamma\left(T \stackrel{*}{E}, \tau_{E}^{*}, \stackrel{*}{E}\right)$, the complete $(\stackrel{*}{g}, h)$-lift of $\stackrel{*}{u}$, satisfying the following conditions:

i) $\stackrel{*^{c}}{u}$ is $(h \circ \stackrel{*}{\pi})$-related with $\Gamma(T h \circ \rho, h \circ \eta)(\Gamma(\stackrel{*}{g}, h)(\stackrel{*}{u}))$, i.e.,

$$
T(h \circ \stackrel{*}{\pi})\left(\stackrel{* c}{u_{v_{x}}^{*}}\right)=\{\Gamma(T h \circ \rho, h \circ \eta)(\Gamma(\stackrel{*}{g}, h)(\stackrel{*}{u}))\}\left(h \circ \stackrel{*}{\pi}\left(\stackrel{*}{v}_{x}\right)\right),
$$

ii) $\stackrel{*^{c}}{u}(\widehat{*})=(\stackrel{*}{g}, \widehat{h}) \mathcal{L}_{*}^{*} * *$

for any $\stackrel{*}{\omega} \in \Lambda^{1}(\stackrel{*}{E}, \stackrel{*}{\pi}, M)$.

Proof. Similar to the proof of Theorem 3.5 , we obtain the following locally expression for $\stackrel{*^{c}}{u}$ that show the existence and uniqueness of it.

$$
\stackrel{*}{u}^{c}=\left(\left(g^{\alpha e} u_{e} \rho_{\alpha}^{i} \circ h\right) \circ \stackrel{*}{\pi}\right) \stackrel{*}{\partial}_{i}-U_{a}\left(K^{\gamma a}(\stackrel{*}{u}) \circ h \circ \stackrel{*}{\pi}\right)\left(\tilde{g}_{b \gamma} \circ \stackrel{*}{\pi}\right) \dot{\partial}^{b},
$$

where

$$
K^{\gamma a}(\stackrel{*}{u})=\left(g^{\beta c} u_{c} \rho_{\beta}^{j} \frac{\partial\left(g^{\gamma a}\right)}{\partial x^{i}}-g^{\alpha a} \rho_{\alpha}^{i} \frac{\partial\left(g^{\gamma c} u_{c}\right)}{\partial x^{i}}+g^{\alpha c} u_{c} L_{\alpha \beta}^{\gamma} g^{\beta a}\right) \circ h^{-1} .
$$

Definition 4.8. The complete $(\stackrel{*}{g}, h)$-lift of a function $f \in \mathcal{F}(N)$ into $\mathcal{F}(\stackrel{*}{E})$ is the function

$$
f^{c}: \stackrel{*}{E} \longrightarrow \mathbb{R}
$$

defined by

$$
\left.f^{c}\right|_{\pi^{*-1}(V)}=\left.U_{a}\left(g^{\alpha a} \circ \stackrel{*}{\pi}\right)\left(\rho_{\alpha}^{i} \circ h \circ \stackrel{*}{\pi}\right) \stackrel{*}{\partial}(f \circ h \circ \stackrel{*}{\pi})\right|_{\pi^{-1}(V)},
$$

where $\left(V, s_{V}\right)$ is an arbitrary vector local $(m+r)$-chart.

Similar to the Lemmas 3.7 and 3.8 , we have

Lemma 4.9. If $\stackrel{*}{u}$ is a section of $(\stackrel{*}{E}, \stackrel{*}{\pi}, M)$ and $f, f_{1}, f_{2} \in \mathcal{F}(N)$, then

$$
\begin{aligned}
& \text { (i) }\left(f_{1}+f_{2}\right)^{c}=f_{1}^{c}+f_{2}^{c}, \\
& (i i)\left(f_{1} f_{2}\right)^{c}=f_{1}^{c} f_{2}^{\vee}+f_{1}^{\vee} f_{2}^{c}, \\
& (\text { iii }) \stackrel{* \vee}{u}\left(f^{c}\right)=\left\{\Gamma(T h \circ \rho, h \circ \eta)\left(\Gamma\left(\stackrel{*}{g}^{*} h\right) \stackrel{*}{*}\right)(f)\right\}^{\vee}, \\
& \text { (iv) } \stackrel{*}{u}^{c}\left(f^{c}\right)=\left\{\left(\Gamma(T h \circ \rho, h \circ \eta)\left(\Gamma\left(\stackrel{*}{g}^{*}, h\right) \stackrel{*}{u}^{*}\right)(f)\right\}^{c} .\right.
\end{aligned}
$$

Definition 4.10. The complete $(\stackrel{*}{g}, h)$-lift $\stackrel{* C}{u}$ of a section $\stackrel{*}{u} \in \Gamma(\stackrel{*}{E}, \stackrel{*}{\pi}, M)$ is the section of $\left((\rho, \eta) T \stackrel{*}{E},(\rho, \eta) \tau_{E}^{*}, \stackrel{*}{E}\right)$ given by

$$
\stackrel{*}{u}^{C}=\left(\left(g^{\alpha e} \circ *\right)\left(u_{e} \circ *\right)\right) \tilde{\partial}_{\alpha}-U_{a}\left(K^{\gamma a}(\stackrel{*}{u}) \circ h \circ \pi^{*}\right)\left(\tilde{g}_{b \gamma} \circ *\right) \dot{\tilde{\partial}}^{b} .
$$


It is easy to check that $\Gamma\left(\stackrel{*}{\tilde{\rho}}, I d_{E}\right)(\stackrel{*}{u})=\stackrel{*}{u}^{c}$.

Definition 4.11. If $\stackrel{*}{u}=u_{a} s^{a}$ be a section of $(\stackrel{*}{E}, \stackrel{*}{\pi}, M)$, then we introduce the vertical lift of $\stackrel{*}{u}$ as section of $\left((\rho, \eta) T \stackrel{*}{E},(\rho, \eta) \tau_{E}^{*}, \stackrel{*}{E}\right)$ given by

$$
\stackrel{*}{u}^{V}=0_{(h \circ \pi) * E} \oplus \stackrel{* \vee}{u} .
$$

If $u=u^{a} e_{a} \in \Gamma(E, \pi, M)$, then in the locally expressions we get

$$
\stackrel{*}{u}^{V}=\left(u_{a} \circ{ }^{*}\right) \dot{\tilde{\partial}}^{a},
$$

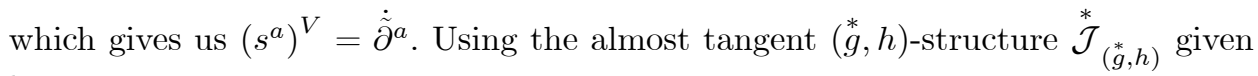
by

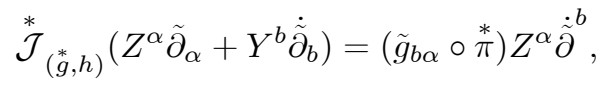

it results that $\stackrel{*}{\mathcal{J}}_{(\stackrel{*}{g}, h)}(\stackrel{*}{u})=\stackrel{*}{u}^{V}$. Similar to Theorem 3.12 we can deduce the following

Theorem 4.12. The Lie brackets of generalized vertical lifts and generalized complete $(\stackrel{*}{g}, h)$-lifts satisfy the following equalities:
i) $\left[\stackrel{* V}{u}, \stackrel{*}{v}^{V}\right]_{(\rho, \eta) T \stackrel{*}{E}}=0$,
ii) $[\stackrel{* V}{u}, \stackrel{* C}{v}]_{(\rho, \eta) T \stackrel{*}{E}}=\left\{\Gamma\left(\stackrel{*}{g}^{-1}, h^{-1}\right)[\Gamma(\stackrel{*}{g}, h) \stackrel{*}{u}, \Gamma(\stackrel{*}{g}, h) \stackrel{*}{v}]_{F, h}\right\}^{V}$,
iii) $\left[\stackrel{*}{u}, \stackrel{*}{v}^{C}\right]_{(\rho, \eta) T \stackrel{*}{E}}=\left\{\Gamma\left(\stackrel{*}{g}^{-1}, h^{-1}\right)[\Gamma(\stackrel{*}{g}, h) \stackrel{*}{u}, \Gamma(\stackrel{*}{g}, h) \stackrel{*}{v}]_{F, h}\right\}^{C}$.

\section{Duality Between vertical and Complete lifts}

A Lagrange fundamental function on the vector bundle $(E, \pi, M)$ is a function $E \stackrel{L}{\longrightarrow} \mathbb{R}$ which satisfies the following conditions:

$L_{1} . L \circ u \in C^{\infty}(M)$, for any $u \in \Gamma(E, \pi, M) \backslash\{0\}$;

$L_{2}$. $L \circ 0 \in C^{0}(M)$, where 0 means the null section of $(E, \pi, M)$.

If $\left(U, s_{U}\right)$ is a local vector $(m+r)$-chart, then we obtain the following real functions defined on $\pi^{-1}(U)$ :

$$
L_{i}=\frac{\partial L}{\partial x^{i}}, \quad L_{i b}=\frac{\partial^{2} L}{\partial x^{i} \partial y^{b}}, \quad L_{a}=\frac{\partial L}{\partial y^{a}}, \quad L_{a b}=\frac{\partial^{2} L}{\partial y^{a} \partial y^{b}} .
$$

If $L$ is a Lagrange fundamental function such that

$$
\operatorname{rank}\left\|L_{a b}\left(u_{x}\right)\right\|=r,
$$


for any $u_{x} \in \pi^{-1}(U) \backslash\left\{0_{x}\right\}$, then we will say that the Lagrange fundamental function $L$ is regular and we obtain the real functions $\tilde{L}^{a b}$ locally defined by

$$
\begin{array}{ccc}
\pi^{-1}(U) & \stackrel{\tilde{L}^{a b}}{\longrightarrow} & \mathbb{R} \\
u_{x} & \longmapsto & \tilde{L}^{a b}\left(u_{x}\right)
\end{array},
$$

where $\left\|\tilde{L}^{a b}\left(u_{x}\right)\right\|=\left\|L_{b a}\left(u_{x}\right)\right\|^{-1}$, for any $u_{x} \in \pi^{-1}(U) \backslash\left\{0_{x}\right\}$ (see [2], for more details).

Definition 5.1. If $L$ is a Lagrange fundamental function, then we build the Legendre bundles morphism

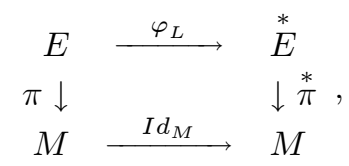

locally defined

$$
\begin{array}{ccc}
\pi^{-1}(U) & \stackrel{\varphi_{L}}{\longrightarrow} & \stackrel{*}{\pi}^{-1}(U), \\
u_{x}=u^{a}(x) s_{a}(x) & \longmapsto & u^{a}(x) L_{a b}\left(u_{x}\right) s^{b}(x),
\end{array}
$$

for any vector local $(m+r)$-charts $\left(U, s_{U}\right)$ and $\left(U, \stackrel{*}{s}_{U}\right)$ of $(E, \pi, M)$ and $\left(\stackrel{*}{E}, * *{ }^{*}, M\right)$, respectively.

Using the above definition, we deduce that if $u=u^{a} s_{a}$ belongs to $\Gamma(E, \pi, M)$, then we obtain its Legendre transformation

$$
\Gamma\left(\varphi_{L}, I d_{M}\right)(u)=\left(u^{a}\left(L_{a b} \circ u\right)\right) s^{b},
$$

belongs to $\Gamma(\stackrel{*}{E}, \stackrel{*}{\pi}, M)$.

If $L$ is a Lagrange fundamental function positively homogenous of degree two, namely

$F_{1}$. $L$ is positively 2 -homogeneous on the fibres of vector bundle $(E, \pi, M)$;

$F_{2}$. For any vector local $(m+r)$-chart $\left(U, s_{U}\right)$ of $(E, \pi, M)$, the Hessian

$$
\left\|L_{a b}\left(u_{x}\right)\right\| \text {, }
$$

is positively define for any $u_{x} \in \pi^{-1}(U) \backslash\left\{0_{x}\right\}$, then $L$ will be called Finsler fundamental function.

Proposition 5.2. If $L$ is a Finsler fundamental function on the vector bundle $(E, \pi, M)$, then

$$
\varphi_{L}\left(u_{x}\right)=L_{b}\left(u_{x}\right) s^{b}(x), \forall u_{x} \in E .
$$

Proof. From 5.1 we have $\varphi_{L}\left(u_{x}\right)=u^{a}(x) L_{a b}\left(u_{x}\right) s^{b}(x)$. But, the Finsler fundamental function $L$ satisfies

$$
u^{a}(x) L_{a b}\left(u_{x}\right)=L_{b},
$$


because $L$ is positively 2 -homogenous on the fibres of $(E, \pi, M)$. This complete the proof.

A Hamilton fundamental function on the dual vector bundle $(\stackrel{*}{E}, \stackrel{*}{\pi}, M)$ is a function $\stackrel{*}{E} \stackrel{H}{\longrightarrow} \mathbb{R}$ which satisfies the following conditions:

$H_{1} . H \circ \stackrel{*}{u} \in C^{\infty}(M)$, for any $\stackrel{*}{u} \in \Gamma(\stackrel{*}{E}, \stackrel{*}{\pi}, M) \backslash\{0\}$;

$H_{2} . H \circ 0 \in C^{0}(M)$, where 0 means the null section of $(\stackrel{*}{E}, \stackrel{*}{\pi}, M)$.

If $\left(U,{ }^{*}{ }_{U}\right)$ is a local vector $(m+r)$-chart, then we obtain the following real functions defined on $\stackrel{*}{\pi}^{-1}(U)$ :

$$
H_{i}=\frac{\partial H}{\partial x^{i}}, \quad H_{i}^{b}=\frac{\partial^{2} H}{\partial x^{i} \partial p_{b}}, \quad H^{a}=\frac{\partial H}{\partial p_{a}}, \quad H^{a b}=\frac{\partial^{2} H}{\partial p_{a} \partial p_{b}} .
$$

If $H$ is a Hamilton fundamental function such that

$$
\operatorname{rank}\left\|H^{a b}\left(\stackrel{*}{u}_{x}\right)\right\|=r,
$$

for any $\stackrel{*}{u}_{x} \in \stackrel{*}{\pi}^{-1}(U) \backslash\left\{0_{x}\right\}$, then we will say that the Hamilton fundamental function $H$ is regular and we obtain the real functions $\tilde{H}_{a b}$ locally defined by

$$
\begin{array}{ccc}
\stackrel{*}{\pi}^{-1}(U) & \stackrel{\tilde{H}_{a b}}{\longrightarrow} & \mathbb{R} \\
\stackrel{*}{u}_{x} & \longmapsto & \tilde{H}_{a b}\left({ }^{*} u_{x}\right)
\end{array},
$$

where $\left\|\tilde{H}_{a b}\left(\stackrel{*}{u}_{x}\right)\right\|=\left\|H^{a b}\left(\stackrel{*}{u}_{x}\right)\right\|^{-1}$, for any $\stackrel{*}{u}_{x} \in \stackrel{*}{\pi}^{-1}(U) \backslash\left\{0_{x}\right\}$.

Definition 5.3. If $H$ is a Hamilton fundamental function on the vector bundle $(\stackrel{*}{E}, \stackrel{*}{\pi}, M)$, then we build the Legendre bundles morphism

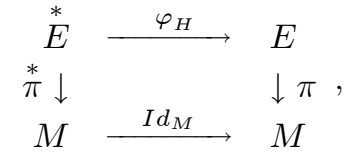

where $\varphi_{H}$ is locally defined

$$
\begin{aligned}
& \stackrel{*}{\pi}^{-1}(U) \stackrel{\varphi_{H}}{\longrightarrow} \quad \pi^{-1}(U) \\
& \stackrel{*}{u}_{x}=u_{a}(x) s^{a}(x), \quad \longmapsto \quad u_{a}(x) H^{a b}\left(\stackrel{*}{u}_{x}\right) s_{b}(x),
\end{aligned}
$$

for any vector local $(m+r)$-chart $\left(U, s_{U}\right)$ of $(E, \pi, M)$ and for any vector local $(m+r)-\operatorname{chart}\left(U, \stackrel{*}{s}_{U}\right)$ of $(\stackrel{*}{E}, \stackrel{*}{\pi}, M)$.

Using the above definition, we deduce that if $\stackrel{*}{u}=u_{a} s^{a}$ belongs to $\Gamma(\stackrel{*}{E}, \stackrel{*}{\pi}, M)$, then we obtain its Legendre transformation

$$
\Gamma\left(\varphi_{H}, I d_{M}\right)(\stackrel{*}{u})=\left(u_{a}\left(H^{a b} \circ u\right)\right) s_{b},
$$

belongs to $\Gamma(E, \pi, M)$. 
Definition 5.4. If $H$ is Hamilton fundamental function positively homogeneous of degree two, namely

$C_{1} . H$ is positively 2-homogeneous on the fibres of vector bundle $(\stackrel{*}{E}, \stackrel{*}{\pi}, M)$;

$C_{2}$. For any vector local $(m+r)$-chart $\left(U, \stackrel{*}{s}_{U}\right)$ of $(\stackrel{*}{E}, \stackrel{*}{\pi}, M)$, the Hessian:

$$
\left\|H^{a b}\left(\stackrel{*}{u}_{x}\right)\right\|,
$$

is positively define for any $\stackrel{*}{u}_{x} \in \stackrel{*}{\pi}^{-1}(U) \backslash\left\{0_{x}\right\}$, then $H$ will be called Cartan fundamental function.

Similar to Proposition 5.2. we have the following

Proposition 5.5. If $H$ is a Cartan fundamental function, then

$$
\varphi_{H}\left(\stackrel{*}{u}_{x}\right)=H^{b}\left(\stackrel{*}{u}_{x}\right) s_{b}(x), \forall \stackrel{*}{u}_{x} \in \stackrel{*}{E} .
$$

Theorem 5.6. If $L$ is a Lagrange fundamental function on the vector bundle $(E, \pi, M)$ and $H$ is a Hamiltonian on the dual vector bundle $(\stackrel{*}{E}, \stackrel{*}{\pi}, M)$, then

i) $\varphi_{H} \circ \varphi_{L}=I d_{\pi^{-1}(U)}$ if and only if $L$ is regular and $\tilde{L}^{a b}=H^{a b} \circ \varphi_{L}$,

ii) $\varphi_{L} \circ \varphi_{H}=I d_{\pi^{-1}(U)}$ if and only if $H$ is regular and $\tilde{H}_{a b}=L_{a b} \circ \varphi_{H}$.

Proof. Using Definition 5.1 and Definition 5.3 , we deduce that

$$
\begin{aligned}
\varphi_{H} \circ \varphi_{L}\left(u_{x}\right) & =\varphi_{H}\left(u^{a}(x) L_{a b}\left(u_{x}\right) s^{b}(x)\right) \\
& =u^{a}(x) L_{a b}\left(u_{x}\right) H^{b c}\left(\varphi_{L}\left(u_{x}\right)\right) s_{c}(x) \\
& =I d_{\pi^{-1}(U)}\left(u_{x}\right),
\end{aligned}
$$

if and only if

$$
L_{a b}\left(u_{x}\right) H^{b c}\left(\varphi_{L}\left(u_{x}\right)\right)=\delta_{a}^{c}\left(u_{x}\right),
$$

for any $u_{x} \in \pi^{-1}(U)$. Thus we have (i). Similar, we can prove (ii).

Definition 5.7. If $L$ is a Lagrange fundamental function on the vector bundle $(E, \pi, M)$, then the Hamilton fundamental function $H$, locally defined by

$$
\begin{array}{ccc}
\stackrel{*}{\pi}^{-1}(U) & \stackrel{H}{ } & \mathbb{R} \\
\stackrel{*}{u}_{x}=u_{a}(x) s^{a}(x) & \longmapsto & u_{a}(x) u^{a}(x)-L\left(u_{x}\right)
\end{array},
$$

for any vector local $(m+r)$-chart $\left(U \stackrel{*}{s}_{U}\right)$ of $(\stackrel{*}{E}, \stackrel{*}{\pi}, M)$, where $u^{a}(x), a \in\{1, \cdots, r\}$, are the components of the solution of the system of differentiable equations

$$
\left\{\begin{array}{ccc}
u_{1}(x) & = & u^{a}(x) L_{a 1}\left(u_{x}\right), \\
\vdots & \vdots & \vdots \\
u_{r}(x) & = & u^{a}(x) L_{a r}\left(u_{x}\right),
\end{array}, u_{x} \in \pi^{-1}(U),\right.
$$

will be called the Legendre transformation of the Lagrangian $L$. 
It is remarkable that in the general case, if $L$ is a Lagrange fundamental function on the vector bundle $(E, \pi, M)$ and $H$ is its Legendre transformation, then $H \circ \varphi_{L} \neq$ $L$, but in particular, if $L$ is a Finsler fundamental function on the vector bundle $(E, \pi, M)$ and $H$ is its Legendre transformation, then $H \circ \varphi_{L}=L$.

Definition 5.8. If $H$ is a Hamilton fundamental function on the dual vector bundle $(\stackrel{*}{E}, * *, M)$, then the Lagrange fundamental function $L$, locally defined by

$$
\begin{array}{ccc}
\pi^{-1}(U) & \stackrel{L}{\longrightarrow} & \mathbb{R} \\
u_{x}=u^{a}(x) s_{a}(x) & \longmapsto & u^{a}(x) u_{a}(x)-H\left({ }^{*} u_{x}\right)
\end{array},
$$

for any vector local $(m+r)$-chart $\left(U, s_{U}\right)$ of $(E, \pi, M)$, where $\left(u_{a}(x), a \in \overline{1, r}\right)$ are the components of the solution of the system of differentiable equations

$$
\left\{\begin{array}{ccc}
u^{1}(x) & = & u_{a}(x) H^{a 1}\left(\stackrel{*}{u}_{x}\right) \\
\vdots & \vdots & \vdots \\
u^{r}(x) & = & u_{a}(x) H^{a r}\left(\stackrel{*}{u}_{x}\right)
\end{array}, \stackrel{*}{u}_{x} \in \stackrel{*}{\pi}^{-1}(U)\right.
$$

will be called the Legendre transformation of the Hamiltonian $H$.

In general, if $H$ is a Hamilton fundamental function on the vector bundle $(\stackrel{*}{E}, * *, M)$ and $L$ is its Legendre transformation, then $L \circ \varphi_{H} \neq H$, but in particular, if $H$ is a Cartan fundamental function on the vector bundle $(\stackrel{*}{E}, \stackrel{*}{\pi}, M)$ and $L$ is its Legendre transformation, then $L \circ \varphi_{H}=H$.

Remark 5.9. The Hamilton fundamental function $H$ is the Legendre transformation of the Lagrange fundamental function $L$ if and only if the Lagrange fundamental function $L$ is the Legendre transformation of the Hamilton fundamental function $H$.

Let $L$ be a Lagrangian on the vector bundle $(E, \pi, M)$ and let $H$ be its Legendre transformation.

Using the Legendre bundles morphism $\left(\varphi_{L}, I d_{M}\right)$, we build the vector bundles morphism $\left((\rho, \eta) T \varphi_{L}, \varphi_{L}\right)$ given by the diagram

$$
\begin{array}{rll}
(\rho, \eta) T E & \stackrel{(\rho, \eta) T \varphi_{L}}{\longrightarrow} & (\rho, \eta) T \stackrel{*}{E} \\
(\rho, \eta) \tau_{E} \downarrow & & \downarrow(\rho, \eta) \tau_{E}^{*} \\
E & \stackrel{\varphi_{L}}{\longrightarrow} & \stackrel{*}{E}
\end{array}
$$

such that

$$
\begin{aligned}
& \Gamma\left((\rho, \eta) T \varphi_{L}, \varphi_{L}\right)\left(Z^{\alpha} \tilde{\partial}_{\alpha}\right)=\left(Z^{\alpha} \circ \varphi_{H}\right) \dot{\tilde{\partial}}_{\alpha}+\left[\left(\rho_{\alpha}^{i} \circ h \circ \pi\right) Z^{\alpha} L_{i b}\right] \circ \varphi_{H} \dot{\tilde{\partial}}^{b}, \\
& \Gamma\left((\rho, \eta) T \varphi_{L}, \varphi_{L}\right)\left(Y^{a} \dot{\tilde{\partial}}_{a}\right)=\left(Y^{a} L_{a b}\right) \circ \varphi_{H} \tilde{\tilde{\partial}}^{b}
\end{aligned}
$$

for any $Z^{\alpha} \tilde{\partial}_{\alpha}+Y^{a} \dot{\tilde{\partial}}_{a} \in \Gamma\left((\rho, \eta) T E,(\rho, \eta) \tau_{E}, E\right)$. The vector bundles morphism $\left((\rho, \eta) T \varphi_{L}, \varphi_{L}\right)$ will be called the tangent $(\rho, \eta)$-application of the Legendre bundles 
morphism associated to the Lagrangian L. Using this application together with (3.5), 3.6 we deduce the following theorems.

Theorem 5.10. If $u=u^{a} s_{a} \in \Gamma(E, \pi, M)$ such that

$$
\Gamma\left((\rho, \eta) T \varphi_{L}, \varphi_{L}\right)\left(u^{V}\right)=\Gamma\left(\varphi_{L}, I d_{M}\right)(u),
$$

then

$$
u^{a} \circ \pi \circ \varphi_{H}=u^{a} \circ \stackrel{*}{\pi}, \quad L_{a b} \circ \varphi_{H}=L_{a b} \circ u \circ \stackrel{*}{\pi} .
$$

Theorem 5.11. If $u=u^{a} s_{a} \in \Gamma(E, \pi, M)$ such that

$$
\Gamma\left((\rho, \eta) T \varphi_{L}, \varphi_{L}\right)\left(u^{C}\right)=\Gamma\left(\varphi_{L}, I d_{M}\right)(u)
$$

then

$$
\begin{aligned}
\left(g^{\alpha e} u_{e}\right) \circ \stackrel{*}{\pi}= & \left(g_{e}^{\alpha} u^{e}\right) \circ \pi \circ \varphi_{H}, \\
U_{a}\left[K^{\gamma a}(\stackrel{*}{u}) \circ h \circ \stackrel{*}{\pi}\right]\left(\tilde{g}_{b \gamma} \circ \stackrel{*}{\pi}\right)= & \left\{U^{a}\left(K_{a}^{\gamma}(u) \circ h \circ \pi\right)\left(\tilde{g}_{\gamma}^{c} \circ \pi\right) L_{c b}\right\} \circ \varphi_{H} \\
& -\left\{\left(\rho_{\alpha}^{i} \circ h \circ \pi\right)\left(\left(g_{e}^{\alpha} u^{e}\right) \circ \pi\right) L_{i b}\right\} \circ \varphi_{H} .
\end{aligned}
$$

Using the bundles morphism $\left(\varphi_{H}, I d_{M}\right)$, we build the vector bundles morphism $\left((\rho, \eta) T \varphi_{H}, \varphi_{H}\right)$ given by the diagram

$$
\begin{array}{ccl}
(\rho, \eta) T E^{*} & \stackrel{(\rho, \eta) T \varphi_{H}}{\longrightarrow} & (\rho, \eta) T E \\
(\rho, \eta) \tau_{E}^{*} \downarrow & & \downarrow(\rho, \eta) \tau_{E}, \\
\varphi_{E^{*}} & \varphi_{H} &
\end{array}
$$

such that

$$
\begin{aligned}
\Gamma\left((\rho, \eta) T \varphi_{H}, \varphi_{H}\right)\left(Z^{\alpha} \tilde{\partial}_{\alpha}^{*}\right) & =\left(Z^{\alpha} \circ \varphi_{L}\right) \tilde{\partial}_{\alpha}+\left[\left(\rho_{\alpha}^{i} \circ h \circ \pi^{*}\right) Z^{\alpha} H_{i}^{b}\right] \circ \varphi_{L} \dot{\tilde{\partial}}_{b}, \\
\Gamma\left((\rho, \eta) T \varphi_{H}, \varphi_{H}\right)\left(Y_{a} \dot{\tilde{\partial}}^{a}\right) & =\left(Y_{a} H^{a b}\right) \circ \varphi_{L} \dot{\tilde{\partial}}_{b},
\end{aligned}
$$

for any $Z^{\alpha} \stackrel{*}{\partial}_{\alpha}+Y_{a} \stackrel{\cdot}{\tilde{\partial}}^{a} \in \Gamma\left((\rho, \eta) T \stackrel{*}{E},(\rho, \eta) \tau_{E}^{*}, \stackrel{*}{E}\right)$. The vector bundles morphism $\left((\rho, \eta) T \varphi_{H}, \varphi_{H}\right)$ will be called the tangent $(\rho, \eta)$-application of the Legendre bundles morphism associated to the Hamiltonian $H$. Using this application together with (4.1) and 4.2 we deduce the following theorems.

Theorem 5.12. If $\stackrel{*}{u}=u_{a} s^{a} \in \Gamma(\stackrel{*}{E}, \stackrel{*}{\pi}, M)$ such that

$$
\Gamma\left((\rho, \eta) T \varphi_{H}, \varphi_{H}\right)\left(\stackrel{*}{u}^{V}\right)=\Gamma\left(\varphi_{H}, I d_{M}\right)\left({ }_{u}^{*}\right),
$$

then

$$
u_{a} \circ \stackrel{*}{\pi} \circ \varphi_{L}=u_{a} \circ \pi, \quad H^{a b} \circ \varphi_{L}=H^{a b} \circ \stackrel{*}{u} \circ \pi .
$$

Theorem 5.13. If $\stackrel{*}{u}=u_{a} s^{a} \in \Gamma(\stackrel{*}{E}, \stackrel{*}{\pi}, M)$ such that

$$
\Gamma\left((\rho, \eta) T \varphi_{H}, \varphi_{H}\right)\left({ }^{*}{ }^{C}\right)=\Gamma\left(\varphi_{H}, I d_{M}\right)\left({ }_{u}^{*}\right),
$$


then

$$
\begin{aligned}
\left(g_{e}^{\alpha} u^{e}\right) \circ \pi= & \left(g^{\alpha e} u_{e}\right) \circ \pi^{*} \circ \varphi_{L}, \\
U^{a}\left(K_{a}^{\gamma}(u) \circ h \circ \pi\right)\left(\tilde{g}_{\gamma}^{b} \circ \pi\right)= & \left\{U_{a}\left(K^{\gamma a}\left(\stackrel{*}{u} \circ \circ \circ \pi^{*}\right)\left(\tilde{g}_{c \gamma} \circ \pi^{*}\right) H^{c b}\right\} \circ \varphi_{L}\right. \\
& -\left\{\left(\rho_{\alpha}^{i} \circ h \circ *\right)\left(\left(g_{e}^{\alpha} u^{e}\right) \circ *\right) H_{i}^{b}\right\} \circ \varphi_{L} .
\end{aligned}
$$

6. Duality Between Lie algebroids structures

Theorem 6.1. If the vector bundles morphism $\left((\rho, \eta) T \varphi_{L}, \varphi_{L}\right)$ is a morphism of Lie algebroids, then we obtain:

$$
\begin{gathered}
\left(L_{\alpha \beta}^{\gamma} \circ h \circ \pi\right) \circ \varphi_{H}=L_{\alpha \beta}^{\gamma} \circ h \circ \stackrel{*}{ }, \\
\left(\left(L_{\alpha \beta}^{\gamma} \rho_{\gamma}^{k}\right) \circ h \circ \pi \cdot L_{k b}\right) \circ \varphi_{H}=\rho_{\alpha}^{i} \circ h \circ \pi \cdot \frac{\partial}{\partial x^{i}}\left(\left(\rho_{\beta}^{j} \circ h \circ \pi \cdot L_{j b}\right) \circ \varphi_{H}\right) \\
-\rho_{\beta}^{j} \circ h \circ \pi \cdot \frac{\partial}{\partial x^{j}}\left(\left(\rho_{\alpha}^{i} \circ h \circ \pi \cdot L_{i b}\right) \circ \varphi_{H}\right) \\
+\left(\rho_{\alpha}^{i} \circ h \circ \pi \cdot L_{i a}\right) \circ \varphi_{H} \cdot \frac{\partial}{\partial p_{a}}\left(\left(\rho_{\beta}^{j} \circ h \circ \pi \cdot L_{j b}\right) \circ \varphi_{H}\right) \\
-\left(\rho_{\beta}^{j} \circ h \circ \pi \cdot L_{j a}\right) \circ \varphi_{H} \cdot \frac{\partial}{\partial p_{a}}\left(\left(\rho_{\alpha}^{i} \circ h \circ \pi \cdot L_{i b}\right) \circ \varphi_{H}\right), \\
0=\rho_{\alpha}^{i} \circ h \circ \pi \cdot \frac{\partial}{\partial x^{i}}\left(L_{b a} \circ \varphi_{H}\right)+\left(\rho_{\alpha}^{i} \circ h \circ \pi \cdot L_{b c}\right) \circ \varphi_{H} \frac{\partial}{\partial p_{c}}\left(L_{i a} \circ \varphi_{H}\right) \\
-L_{b c} \circ \varphi_{H} \cdot \frac{\partial}{\partial p_{c}}\left(\left(\rho_{\alpha}^{i} \circ h \circ \pi \cdot L_{i a}\right) \circ \varphi_{H}\right),
\end{gathered}
$$

and

$$
0=L_{a c} \circ \varphi_{H} \cdot \frac{\partial}{\partial p_{c}}\left(L_{b d} \circ \varphi_{H}\right)-L_{b d} \circ \varphi_{H} \cdot \frac{\partial}{\partial p_{d}}\left(L_{a c} \circ \varphi_{H}\right) .
$$

Proof. Developing the following equalities

$$
\begin{aligned}
& \Gamma\left((\rho, \eta) T \varphi_{L}, \varphi_{L}\right)\left[\tilde{\partial}_{\alpha}, \tilde{\partial}_{\beta}\right]_{(\rho, \eta) T E} \\
& =\left[\Gamma\left((\rho, \eta) T \varphi_{L}, \varphi_{L}\right) \tilde{\partial}_{\alpha}, \Gamma\left((\rho, \eta) T \varphi_{L}, \varphi_{L}\right) \tilde{\partial}_{\beta}\right]_{(\rho, \eta) T E}, \\
& \Gamma\left((\rho, \eta) T \varphi_{L}, \varphi_{L}\right)\left[\tilde{\partial}_{\alpha}, \dot{\tilde{\partial}}_{b}\right]_{(\rho, \eta) T E} \\
& =\left[\Gamma\left((\rho, \eta) T \varphi_{L}, \varphi_{L}\right) \tilde{\partial}_{\alpha}, \Gamma\left((\rho, \eta) T \varphi_{L}, \varphi_{L}\right) \dot{\tilde{\partial}}_{b}\right]_{(\rho, \eta) T E},
\end{aligned}
$$

and

$$
\begin{aligned}
& \Gamma\left((\rho, \eta) T \varphi_{L}, \varphi_{L}\right)\left[\dot{\tilde{\partial}}_{a}, \dot{\tilde{\partial}}_{b}\right]_{(\rho, \eta) T E} \\
& =\left[\Gamma\left((\rho, \eta) T \varphi_{L}, \varphi_{L}\right) \dot{\tilde{\partial}}_{a}, \Gamma\left((\rho, \eta) T \varphi_{L}, \varphi_{L}\right) \dot{\tilde{\partial}}_{b}\right]_{(\rho, \eta) T E}^{*},
\end{aligned}
$$

it results the conclusion of the theorem.

Corollary 6.2. In the particular case of Lie algebroids, $(\eta, h)=\left(I d_{M}, I d_{M}\right)$, we obtain:

$$
\left(L_{\alpha \beta}^{\gamma} \circ \pi\right) \circ \varphi_{H}=L_{\alpha \beta}^{\gamma} \circ \stackrel{*}{\pi},
$$




$$
\begin{aligned}
& \left(\left(L_{\alpha \beta}^{\gamma} \rho_{\gamma}^{k}\right) \circ \pi \cdot L_{k b}\right) \circ \varphi_{H}=\rho_{\alpha}^{i} \circ * \cdot \frac{\partial}{\partial x^{i}}\left(\left(\rho_{\beta}^{j} \circ \pi \cdot L_{j b}\right) \circ \varphi_{H}\right) \\
& -\rho_{\beta}^{j} \circ \pi \cdot \frac{\partial}{\partial x^{j}}\left(\left(\rho_{\alpha}^{i} \circ \pi \cdot L_{i b}\right) \circ \varphi_{H}\right) \\
& +\left(\rho_{\alpha}^{i} \circ \pi \cdot L_{i a}\right) \circ \varphi_{H} \cdot \frac{\partial}{\partial p_{a}}\left(\left(\rho_{\beta}^{j} \circ \pi \cdot L_{j b}\right) \circ \varphi_{H}\right) \\
& -\left(\rho_{\beta}^{j} \circ \pi \cdot L_{j a}\right) \circ \varphi_{H} \cdot \frac{\partial}{\partial p_{a}}\left(\left(\rho_{\alpha}^{i} \circ \pi \cdot L_{i b}\right) \circ \varphi_{H}\right), \\
0= & \rho_{\alpha}^{i} \circ * \cdot \frac{\partial}{\partial x^{i}}\left(L_{b a} \circ \varphi_{H}\right)+\left(\rho_{\alpha}^{i} \circ \pi \cdot L_{b c}\right) \circ \varphi_{H} \frac{\partial}{\partial p_{c}}\left(L_{i a} \circ \varphi_{H}\right) \\
& -L_{b c} \circ \varphi_{H} \cdot \frac{\partial}{\partial p_{c}}\left(\left(\rho_{\alpha}^{i} \circ \pi \cdot L_{i a}\right) \circ \varphi_{H}\right),
\end{aligned}
$$

and

$$
0=L_{a c} \circ \varphi_{H} \cdot \frac{\partial}{\partial p_{c}}\left(L_{b d} \circ \varphi_{H}\right)-L_{b c} \circ \varphi_{H} \cdot \frac{\partial}{\partial p_{c}}\left(L_{a d} \circ \varphi_{H}\right) .
$$

In the classical case, $(\rho, \eta, h)=\left(I d_{T M}, I d_{M}, I d_{M}\right)$, we obtain:

$$
\begin{gathered}
0=\frac{\partial}{\partial x^{i}}\left(\frac{\partial^{2} L}{\partial x^{j} \partial y^{k}} \circ \varphi_{H}\right)-\frac{\partial}{\partial x^{j}}\left(\frac{\partial^{2} L}{\partial x^{i} \partial y^{k}} \circ \varphi_{H}\right) \\
+\frac{\partial^{2} L}{\partial x^{2} \partial y^{h}} \circ \varphi_{H} \cdot \frac{\partial}{\partial p_{h}}\left(\frac{\partial^{2} L}{\partial x^{j} \partial y^{k}} \circ \varphi_{H}\right)-\frac{\partial^{2} L}{\partial x^{j} \partial y^{h}} \circ \varphi_{H} \cdot \frac{\partial}{\partial p_{h}}\left(\frac{\partial^{2} L}{\partial x^{2} \partial y^{k}} \circ \varphi_{H}\right), \\
0=\frac{\partial}{\partial x^{i}}\left(\frac{\partial^{2} L}{\partial y^{j} L y^{k}} \circ \varphi_{H}\right)+\frac{\partial^{2} L}{\partial x^{i} \partial y^{h}} \circ \varphi_{H} \cdot \frac{\partial}{\partial p_{h}}\left(\frac{\partial^{2} L}{\partial y^{j} \partial y^{k}} \circ \varphi_{H}\right) \\
\quad-\frac{\partial^{2} L}{\partial x^{j} \partial y^{h}} \circ \varphi_{H} \cdot \frac{\partial}{\partial p_{h}}\left(\frac{\partial^{2} L}{\partial x^{i} \partial y^{k}} \circ \varphi_{H}\right),
\end{gathered}
$$

and

$$
0=\frac{\partial^{2} L}{\partial y^{i} \partial y^{h}} \circ \varphi_{H} \cdot \frac{\partial}{\partial p_{h}}\left(\frac{\partial^{2} L}{\partial y^{j} \partial y^{k}} \circ \varphi_{H}\right)-\frac{\partial^{2} L}{\partial y^{j} \partial y^{h}} \circ \varphi_{H} \cdot \frac{\partial}{\partial p_{h}}\left(\frac{\partial^{2} L}{\partial y^{i} \partial y^{k}} \circ \varphi_{H}\right) .
$$

Theorem 6.3. If the vector bundles morphism $\left((\rho, \eta) T \varphi_{H}, \varphi_{H}\right)$ is a morphism of Lie algebroids, then we obtain:

$$
\begin{gathered}
\left(L_{\alpha \beta}^{\gamma} \circ h \circ \stackrel{*}{\pi}\right) \circ \varphi_{L}=L_{\alpha \beta}^{\gamma} \circ h \circ \pi, \\
\left(\left(L_{\alpha \beta}^{\gamma} \rho_{\gamma}^{k}\right) \circ h \circ \stackrel{*}{\pi} \cdot H_{k}^{b}\right) \circ \varphi_{L}=\rho_{\alpha}^{i} \circ h \circ \pi \cdot \frac{\partial}{\partial x^{i}}\left(\left(\rho_{\beta}^{j} \circ h \circ \pi^{*} \cdot H_{j}^{b}\right) \circ \varphi_{L}\right) \\
-\rho_{\beta}^{j} \circ h \circ \pi \cdot \frac{\partial}{\partial x^{j}}\left(\left(\rho_{\alpha}^{i} \circ h \circ \pi \cdot H_{i}^{b}\right) \circ \varphi_{L}\right) \\
+\left(\rho_{\alpha}^{i} \circ h \circ \pi \cdot H_{i}^{c}\right) \circ \varphi_{L} \cdot \frac{\partial}{\partial y^{c}}\left(\left(\rho_{\beta}^{j} \circ h \circ \pi \cdot H_{j}^{b}\right) \circ \varphi_{L}\right) \\
-\left(\rho_{\beta}^{j} \circ h \circ \pi \cdot H_{j}^{c}\right) \circ \varphi_{L} \cdot \frac{\partial}{\partial y^{c}}\left(\left(\rho_{\alpha}^{i} \circ h \circ \pi \cdot H_{i}^{b}\right) \circ \varphi_{L}\right), \\
0=\rho_{\alpha}^{i} \circ h \circ \pi \cdot \frac{\partial}{\partial x^{i}}\left(H^{b a} \circ \varphi_{L}\right)+\left(\rho_{\alpha}^{i} \circ h \circ * \pi^{*} \cdot H^{b c}\right) \circ \varphi_{L} \frac{\partial}{\partial y^{c}}\left(H^{b a} \circ \varphi_{L}\right) \\
-H^{b c} \circ \varphi_{L} \cdot \frac{\partial}{\partial y^{c}}\left(\left(\rho_{\alpha}^{i} \circ h \circ \pi \cdot H_{i}^{a}\right) \circ \varphi_{L}\right),
\end{gathered}
$$

and

$$
0=H^{a c} \circ \varphi_{L} \cdot \frac{\partial}{\partial y^{c}}\left(H^{b d} \circ \varphi_{L}\right)-H^{b c} \circ \varphi_{L} \cdot \frac{\partial}{\partial y^{c}}\left(H^{a d} \circ \varphi_{L}\right) .
$$

Proof. Developing the equalities

$$
\begin{aligned}
& \Gamma\left((\rho, \eta) T \varphi_{H}, \varphi_{H}\right)\left[\stackrel{*}{\tilde{\partial}}_{\alpha}, \stackrel{*}{\tilde{\partial}}_{\beta}\right]_{(\rho, \eta) T E} \\
& =\left[\Gamma\left((\rho, \eta) T \varphi_{H}, \varphi_{H}\right) \stackrel{*}{\tilde{\partial}_{\alpha}}, \Gamma\left((\rho, \eta) T \varphi_{H}, \varphi_{H}\right) \stackrel{*}{\tilde{\partial}_{\beta}}\right]_{(\rho, \eta) T E},
\end{aligned}
$$




$$
\begin{gathered}
\Gamma\left((\rho, \eta) T \varphi_{H}, \varphi_{H}\right)\left[\stackrel{*}{\tilde{\partial}_{\alpha}}, \dot{\tilde{\partial}}^{b}\right]_{(\rho, \eta) T E}^{*} \\
=\left[\Gamma\left((\rho, \eta) T \varphi_{H}, \varphi_{H}\right) \stackrel{*}{\tilde{\partial}}_{\alpha}, \Gamma\left((\rho, \eta) T \varphi_{H}, \varphi_{H}\right) \tilde{\tilde{\partial}}^{b}\right]_{(\rho, \eta) T E},
\end{gathered}
$$

and

$$
\begin{gathered}
\left.\Gamma\left((\rho, \eta) T \varphi_{H}, \varphi_{H}\right)\left[\dot{\tilde{\partial}}^{a}, \dot{\tilde{\partial}}^{b}\right]_{(\rho, \eta) T E}^{*} \dot{\tilde{\tilde{\partial}}}^{b}\right]_{(\rho, \eta) T E}, \\
=\left[\Gamma\left((\rho, \eta) T \varphi_{H}, \varphi_{H}\right) \dot{\tilde{\partial}}^{a}, \Gamma\left((\rho, \eta) T \varphi_{H}, \varphi_{H}\right.\right.
\end{gathered}
$$

it results the conclusion of the theorem.

Corollary 6.4. In the particular case of Lie algebroids, $(\eta, h)=\left(I d_{M}, I d_{M}\right)$, we obtain

$$
\begin{aligned}
& \left(L_{\alpha \beta}^{\gamma} \circ \stackrel{*}{\pi}\right) \circ \varphi_{L}=L_{\alpha \beta}^{\gamma} \circ \pi, \\
& \left(\left(L_{\alpha \beta}^{\gamma} \rho_{\gamma}^{k}\right) \circ \stackrel{*}{\pi} \cdot H_{k}^{b}\right) \circ \varphi_{L}=\rho_{\alpha}^{i} \circ \pi \cdot \frac{\partial}{\partial x^{i}}\left(\left(\rho_{\beta}^{j} \circ * \pi^{*} \cdot H_{j}^{b}\right) \circ \varphi_{L}\right) \\
& -\rho_{\beta}^{j} \circ \pi \cdot \frac{\partial}{\partial x^{j}}\left(\left(\rho_{\alpha}^{i} \circ * \pi \cdot H_{i}^{b}\right) \circ \varphi_{L}\right)+\left(\rho_{\alpha}^{i} \circ * \pi^{*} \cdot H_{i}^{c}\right) \circ \varphi_{L} \cdot \frac{\partial}{\partial y^{c}}\left(\left(\rho_{\beta}^{j} \circ * \pi \cdot H_{j}^{b}\right) \circ \varphi_{L}\right) \\
& -\left(\rho_{\beta}^{j} \circ * \pi \cdot H_{j}^{c}\right) \circ \varphi_{L} \cdot \frac{\partial}{\partial y^{c}}\left(\left(\rho_{\alpha}^{i} \circ * \pi \cdot H_{i}^{b}\right) \circ \varphi_{L}\right), \\
& 0=\rho_{\alpha}^{i} \circ \pi \cdot \frac{\partial}{\partial x^{i}}\left(H^{b a} \circ \varphi_{L}\right)+\left(\rho_{\alpha}^{i} \circ \pi \cdot H^{b c}\right) \circ \varphi_{L} \frac{\partial}{\partial y^{c}}\left(H^{b a} \circ \varphi_{L}\right) \\
& -H^{b c} \circ \varphi_{L} \cdot \frac{\partial}{\partial y^{c}}\left(\left(\rho_{\alpha}^{i} \circ * \pi^{*} \cdot H_{i}^{a}\right) \circ \varphi_{L}\right),
\end{aligned}
$$

and

$$
0=H^{a c} \circ \varphi_{L} \cdot \frac{\partial}{\partial y^{c}}\left(H^{b d} \circ \varphi_{L}\right)-H^{b c} \circ \varphi_{L} \cdot \frac{\partial}{\partial y^{c}}\left(H^{a d} \circ \varphi_{L}\right) .
$$

In the classical case, $(\rho, \eta, h)=\left(I d_{T M}, I d_{M}, I d_{M}\right)$, we obtain

$$
\begin{gathered}
0=\frac{\partial}{\partial x^{i}}\left(\frac{\partial^{2} H}{\partial x^{k} \partial p_{j}} \circ \varphi_{L}\right)-\frac{\partial}{\partial x^{k}}\left(\frac{\partial^{2} H}{\partial x^{i} \partial p_{j}} \circ \varphi_{L}\right)+\frac{\partial^{2} H}{\partial x^{i} \partial p_{h}} \circ \varphi_{L} \cdot \frac{\partial}{\partial y^{h}}\left(\frac{\partial^{2} H}{\partial x^{k} \partial p_{j}} \circ \varphi_{L}\right) \\
-\frac{\partial^{2} H}{\partial x^{k} \partial p_{h}} \circ \varphi_{L} \cdot \frac{\partial}{\partial y^{h}}\left(\left(\frac{\partial^{2} H}{\partial x^{2} \partial p_{j}} \circ \varphi_{L}\right) \circ \varphi_{L}\right), \\
0=\frac{\partial}{\partial x^{k}}\left(\frac{\partial^{2} H}{\partial p_{i} \partial p_{j}} \circ \varphi_{L}\right)+\frac{\partial^{2} H}{\partial p^{2} H p_{h}} \circ \varphi_{L} \cdot \frac{\partial}{\partial y^{h}}\left(\frac{\partial^{2} H}{\partial x_{k} \partial p_{j}} \circ \varphi_{L}\right) \\
\quad-\frac{\partial^{2} H}{\partial p_{j} \partial p_{h}} \circ \varphi_{L} \cdot \frac{\partial}{\partial y^{h}}\left(\frac{\partial^{2} H}{\partial x^{k} \partial p_{i}} \circ \varphi_{L}\right),
\end{gathered}
$$

and

$$
0=\frac{\partial^{2} H}{\partial p_{i} \partial p_{k}} \circ \varphi_{L} \cdot \frac{\partial}{\partial y^{k}}\left(\frac{\partial^{2} H}{\partial p_{j} \partial p_{h}} \circ \varphi_{L}\right)-\frac{\partial^{2} H}{\partial p_{j} \partial p_{k}} \circ \varphi_{L} \cdot \frac{\partial}{\partial y^{k}}\left(\frac{\partial^{2} H}{\partial p_{i} \partial p_{h}} \circ \varphi_{L}\right) .
$$

Definition 6.5. If $\left((\rho, \eta) T \varphi_{L}, \varphi_{L}\right)$ and $\left((\rho, \eta) T \varphi_{H}, \varphi_{H}\right)$ are Lie algebroids morphisms, then we will say that $(E, \pi, M)$ and $(\stackrel{*}{E}, *, \pi, M)$ are Legendre $(\rho, \eta)$-equivalent and we will write

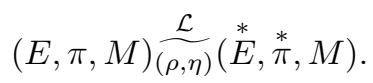

Acknowledgment. This work was supported by Arak University [grant number 93/13595]. 


\section{REFERENCES}

[1] Arcuş, C. M., Generalized Lie algebroids and connections over pair of diffeomorphic manifolds, J. Gen. Lie Theory Appl. 7, (2013), 32 pages.

[2] Arcus, C. M., Mechanical systems in the generalized Lie algebroids framework, Int. J. Geom. Methods Mod. Phys. 11, (2014), 40 pages.

[3] Arcuş, C. M. and Peyghan, E., (Pseudo) generalized Kaluza-Klein G-spaces and Einstein equations, International Journal of Mathematics 25(12), (2014), doi: 10.1142/S0129167X1450116X.

[4] Arcuş, C.M., Peyghan, E. and Sharahi, E., Weyl's theory in the generalized Lie algebroids framework, J. Math Phys. 55, (2014), doi: 10.1063/1.4903256.

[5] Esin, E. and Civelek, S., The lifts on the second order tangent bundles, J. Math. Stat. Fac. Art. Sc. Gazi Univ. 2, (1989), 117-135.

[6] de León M., Marrero J.C., Martínez E.: Lagrangian submanifolds and dynamics on Lie algebroids, J. Phys. A: Math. Gen. 38 (2005), 241-308.

[7] Martínez, E., Lagrangian mechanics on Lie algebroids, Acta Appl. Math. 67, (2001), 295-320.

[8] Omran, T., Sharffuddin, A. and Husain, S.I., Lifts of structures on manifolds, Publications De L'institut Math. 36(50), (1984), 93-97.

[9] Özkan, M., Prolongations of golden structures to tangent bundles, Differential GeometryDynamical Systems 16, (2014), 227-238.

[10] Özkan, M., Çıtlak, A. A. and Taylan E., Prolongations of golden structure to tangent bundle of Order 2, Gazi University Journal of Science 28(2), (2015), 253-258.

[11] Özkan, M. and Yılmaz F., Prolongations of golden structures to tangent bundles of order $r$, Commun. Fac. Sci. Univ. Ank. Ser. A1 Math. Stat. 65(1), (2016), 35-47.

[12] Özkan, M., Taylan, E. and Çıtlak, A. A., On lifts of silver structure, Journal of Science and Arts 2(39), (2017), 223-234.

[13] Peyghan, E., Models of Finsler Geometry on Lie algebroids, arXiv: 1310.7393v1, (2013), 90 pages.

[14] Peyghan, E., Nasrabadi, H. and Tayebi, A., The homogenous lift of the (1, 1)-tensor bundle of a Riemannian metric, Int. J. Geom. Methods Mod. Phys. 10, (2013), 18 pages.

[15] Popescu, L., The geometry of Lie algebroids and its applications to optimal control, arXiv:1302.5212v2 [Math.DG] 25 Feb 2013.

[16] Popescu, L., A note on Poisson-Lie algebroids, J. Geom. Symmetry Phys. 12, (2008), 63-73.

[17] Salimov, A.A and Magden, A., Complete lifts of tensor fields on a pure cross-section in the tensor bundle $T_{q}^{1}\left(M_{n}\right)$, Note di Matematica 18, (1998), 27-37.

[18] Sarlet, W. and Waeyaert, G., Lifting geometric objects to the dual of the first jet bundle of a bundle fibred over $\mathbb{R}, J$. Geom and Phys. 74, (2013), 109-118.

[19] Sasaki, S., On the differential geometry of tangent bundles of Riemannian manifolds, Tohoku Math. J. (I, 10 (1958) 338-354; II, 14 (1962) 146-155).

[20] Yano, K. and Ishihara, S., Tangent and Cotangent Bundles, Marcel Dekker, 1973. 
Current address: E. Peyghan: Department of Mathematics, Faculty of Science, Arak University, Arak, 38156-8-8349, Iran.

E-mail address: e-peyghan@araku.ac.ir

ORCID Address: http://orcid.org/0000-0002-2713-6253

Current address: L. Nourmohammadifar: Department of Mathematics, Faculty of Science, Arak University, Arak, 38156-8-8349, Iran.

E-mail address: 1-nourmohammadifar@phd.araku.ac.ir

ORCID Address: http://orcid.org/0000-0002-8772-4460

Current address: C. M. Arcuş: Secondary School of Cornelius Raduâ, Radinesti Village, 217196 Gorj County, Romania

E-mail address: c_arcus@radinesti.ro

ORCID Address: http://orcid.org/0000-0001-6481-5231 